\title{
Sur les traces de la mémoire seigneuriale au Québec : identité et transmission au sein des familles d'ascendance seigneuriale
}

\section{Benoît Grenier}

Volume 72, numéro 3, hiver 2019

URI : https://id.erudit.org/iderudit/1059979ar

DOI : https://doi.org/10.7202/1059979ar

Aller au sommaire du numéro

\section{Éditeur(s)}

Institut d'histoire de l'Amérique française

\section{ISSN}

0035-2357 (imprimé)

1492-1383 (numérique)

Découvrir la revue

\section{Citer cet article}

Grenier, B. (2019). Sur les traces de la mémoire seigneuriale au Québec :

identité et transmission au sein des familles d'ascendance seigneuriale. Revue d'histoire de l'Amérique française, 72(3), 5-40. https://doi.org/10.7202/1059979ar
Résumé de l'article

Aboli en 1854, le régime seigneurial a laissé une empreinte considérable dans la province de Québec en perpétuant rentes et propriétés seigneuriales. La relation seigneur/censitaire et le mode de vie seigneurial ont persisté jusque tard au XX ${ }^{\mathrm{e}}$ siècle dans bon nombre de communautés. Le présent article s'inscrit dans une recherche sur les persistances seigneuriales dans le Québec contemporain et a pour objectif d'analyser la mémoire seigneuriale. Il émane d'une enquête d'histoire orale menée auprès de familles seigneuriales provenant des quatre coins de la vallée du Saint-Laurent. Il postule l'existence d'une culture familiale distinctive se caractérisant notamment par le maintien de pratiques et d'usages seigneuriaux, d'un rapport d'altérité dans les campagnes québécoises, mais aussi par une mémoire familiale révélant des valeurs seigneuriales portées et transmises par les descendants de ces familles, nobles ou roturières, francophones ou anglophones. 


\title{
Sur les traces de la mémoire seigneuriale au Québec: identité et transmission au sein des familles d'ascendance seigneuriale
}

\author{
BENOÎT GRENIER \\ Université de Sherbrooke
}

RÉsumÉ • Aboli en 1854, le régime seigneurial a laissé une empreinte considérable dans la province de Québec en perpétuant rentes et propriétés seigneuriales. La relation seigneur/ censitaire et le mode de vie seigneurial ont persisté jusque tard au $X X^{e}$ siècle dans bon nombre de communautés. Le présent article s'inscrit dans une recherche sur les persistances seigneuriales dans le Québec contemporain et a pour objectif d'analyser la mémoire seigneuriale. II émane d'une enquête d'histoire orale menée auprès de familles seigneuriales provenant des quatre coins de la vallée du Saint-Laurent. II postule l'existence d'une culture familiale distinctive se caractérisant notamment par le maintien de pratiques et d'usages seigneuriaux, d'un rapport d'altérité dans les campagnes québécoises, mais aussi par une mémoire familiale révélant des valeurs seigneuriales portées et transmises par les descendants de ces familles, nobles ou roturières, francophones ou anglophones.

ABstract - Abolished in 1854, the seigneurial regime left a remarkable imprint in the Province of Quebec by perpetuating rents and seigniorial lands. The seigneur/censitaire relationship and the seigneurial way of life persisted into the late twentieth century in many

1. Ce texte sera suivi d'une seconde partie qui abordera les "porteurs de mémoire» non affiliés aux familles seigneuriales. Je tiens à remercier le Conseil de recherches en sciences humaines du Canada qui a alloué une subvention Savoir à ce projet. Louise Bienvenue, Stéphanie Lanthier, Karine Pépin ainsi que les évaluateurs anonymes de la Revue ont lu et commenté une première version de ce texte. Je les remercie sincèrement pour leur disponibilité et la pertinence de leurs remarques. Des remerciements sincères sont également adressés aux assistants de recherche qui ont collaboré aux diverses étapes du projet depuis 2014: Hubert Cousineau, Jonathan Fortin, Mathieu Lévesque-Dupéré et, surtout, Michel Morissette et Félix-Antoine Têtu. Enfin, un immense merci à Stéphanie Lanthier, collaboratrice essentielle au volet cinématographique de ce projet. Pour voir les documents filmiques réalisés dans le cadre de la recherche: $<$ www.usherbrooke.ca/histoire/recherche/memoires-seigneuriales $>$. 
communities. This article is part of a research on seigneurial persistence in contemporary Quebec and aims to analyze seigneurial memory. It stems from an oral histor"y project with seigneurial families throughout the St. Lawrence Valley. It postulates the existence of a distinctive family culture characterized in particular by a continuance of seigneurial practices and uses, a distinctive sense of otherness in the Quebec countryside, and a family memory revealing seigneurial values brought and transmitted by the descendants of these families, be they nobles or commoners, francophones or anglophones.

Si vous doutez de la survivance des familles nobles [...] je vous les ferai voir continuellement mêlées aux événements marquants de notre vie nationale jusqu'en ces dernières années, et toujours au premier rang en dépit de la poussée qu'ont faite les nouveaux riches en notre pays comme dans les vieilles contrées d'Europe.

Louis-Alexandre Taschereau, «La noblesse canadienne-française», 1922.

\begin{abstract}
Aril 1922: Louis-Alexandre Taschereau, alors premier ministre de la LProvince de Québec, prononce une conférence intitulée «La noblesse canadienne-française». C'est devant un auditoire anglophone à Toronto, dans la langue de Shakespeare, qu'il s'exprime². Sa conférence se révèle un vibrant hommage aux familles nobles qui "sont bien restées à la tête de notre peuple [...] au travail et à l'honneur dans la suite de l'histoire». Taschereau ne se limite pas à montrer leur action aux XVIII ${ }^{\mathrm{e}}$ et $\mathrm{XIX}^{\mathrm{e}}$ siècles, il les présente, nombreux exemples à l'appui, encore influentes à son époque dans les sphères de l'administration publique, de la magistrature et, bien sûr, noblesse oblige, de l'armée ${ }^{3}$. Le premier ministre est toutefois plus discret sur le maintien de la propriété seigneuriale qui caractérise aussi plusieurs de ces familles au début du $\mathrm{XX}^{\mathrm{e}}$ siècle. L’identité nobiliaire constitue une dimension importante des familles seigneuriales du Québec,
\end{abstract}

2. Ce discours prononcé en anglais à Toronto le 27 avril 1922 devant le Women's Canadian Club sera traduit et publié, notamment, dans la Revue moderne en juillet 1930: Louis-Alexandre Taschereau, «La noblesse canadienne-française", La Revue moderne (juillet 1930), p. 9. Il fit d'abord l'objet d'une édition dès 1922, par le journal Le Canada dans un petit livret regroupant les textes traduits de deux conférences prononcées par le Premier ministre ce même jour devant deux auditoires de Toronto.

3. Il garde néanmoins une certaine retenue en ne citant pas sa propre famille, elle-même aux premiers rangs. Pour s'en convaincre: Brian Young, Patrician Families and the Making of Quebec. The Taschereaus and McCord (Montréal et Kingston, McGill-Queen’s University Press, 2014). 
mais celles-ci ne peuvent néanmoins toutes se targuer, comme les Taschereau, d'appartenir au «Second ordre».

Aboli en 1854 par l'Acte pour l'abolition des droits et devoirs féodaux dans le Bas-Canada ${ }^{4}$, le régime seigneurial aura marqué le territoire laurentien pendant plus de deux siècles. Qui plus est, il aura laissé, au moment de sa disparition, des effets bien tangibles sur les principaux intéressés, seigneurs et censitaires, pendant encore près d'un siècle ${ }^{5}$. D'une part, jusqu'en novembre 1940, des rentes constituées continueront d'être versées annuellement, au jour de la Saint-Martin d'hiver ${ }^{6}$, aux détenteurs de droits seigneuriaux - nommément désignés comme seigneurs en vertu même de la loi d'abolition?. Ce groupe diversifié des "derniers seigneurs" du Québec se partagea plus de 3 millions de dollars au cours de la décennie 1940 , en sus des indemnisations reçues au XIX ${ }^{\mathrm{e}}$ siècle ${ }^{8}$. D’autre part, la loi d'abolition aura laissé aux seigneurs la pleine propriété des terres non concédées, contribuant alors à ériger en domaines privés de larges pans du territoire québécois, notamment dans l'est de la province? ${ }^{9}$.

Les propos de Taschereau invitent à considérer les persistances du groupe seigneurial dans la longue durée dans une optique apparentée à l'étude, classique, d'Arno Mayer sur les persistances d'Ancien Régime dans les sociétés européennes ${ }^{10}$. Notre réflexion sur la mémoire et l'identité des familles seigneuriales du Québec fait aussi écho aux travaux d’Éric MensionRigau sur l'adaptation de la noblesse française aux XIX et $\mathrm{XX}^{\mathrm{e}}$ siècles. Dans l'ouvrage issu de sa thèse, il analysait «la transmission depuis la fin du XIX

4. Statuts du Canada, 1854-1855, $1^{\text {re }}$ session, c. 3. [Ci-après "Acte seigneurial»].

5. Nous ne referons pas ici l'historique de l'abolition ni de ses modalités. Voir à ce sujet: Benoît Grenier, “Le dernier endroit dans l'univers” : À propos de l'extinction des rentes seigneuriales au Québec, 1854-1971 ", Revue d'histoire de l'Amérique française, 64, 2 (automne 2010), p. 75-98.

6. Le 11 novembre.

7. L'article XXXVII de la loi d'abolition stipule que les détenteurs et débiteurs de ces rentes constituées continueraient à être légalement appelés « seigneurs et censitaires».

8. Les dernières rentes «seigneuriales» sont officiellement versées le 11 novembre 1940 aux seigneurs québécois. Après cette date, le Syndicat national du rachat des rentes seigneuriales remboursera aux propriétaires de ces rentes (les seigneurs) la valeur capitalisée de ce qui leur restait à percevoir. Les censitaires, quant à eux, continueront à acquitter une "rente" sous forme de taxe municipale, laquelle s'éteindra en 1970. Voir l'analyse de Michel Morissette sur cette question de l'argent: "L'argent et la propriété seigneuriale de 1854 à 1940 : qui sont les gagnants et les perdants du processus d'abolition?», dans Benoît Grenier et Michel Morissette, dir. (avec la coll. d'Alain Laberge et Alex Tremblay-Lamarche), Nouveaux regards en histoire seigneuriale au Québec (Québec, Septentrion, 2016), p. 314-333.

9. Sur la privatisation des terres seigneuriales: Benoît Grenier et Michel Morissette, «Propriétés et propriétaires seigneuriaux dans l'est du Québec entre 1854 et le milieu du XX ${ }^{\mathrm{e}}$ siècle: le cheminement comparé de l'île d'Anticosti et de Rivière-du-Loup", dans Harold Bérubé et Stéphane Savard, dir., Pouvoir et Territoire au Québec depuis 1850 (Québec, Septentrion, 2017), p. 25-60.

10. Arno Mayer, La persistance de l'Ancien Régime. L'Europe de 1848 à la Grande Guerre (Paris, Flammarion, 2010 [1981]). 
siècle, des traditions et des valeurs familiales dans l'aristocratie et dans la grande bourgeoisie [afin] de contribuer à la description sociologique d'un groupe qui appartient de longue date à l'“élite”"11». L'enquête orale menée par ce dernier auprès des châtelains de la France contemporaine est particulièrement révélatrice du maintien à long terme d'un certain nombre d'habitus ${ }^{12}$.

Au Québec, Brian Young demeure cependant l'un des seuls à avoir mis de l'avant ces "vestiges seigneuriaux" postérieurs à l'abolition ${ }^{13}$, notamment par l'étude de l'autorité des familles Taschereau et McCord qu'il observe dans une comparaison de longue durée intergénérationnelle. Il écrit, en introduction de son ouvrage paru en 2014:

Successful dynasties like the McCords and the Taschereaus - presented here as families able to regenerate wealth, status, and authority over generations - are necessarily engaged in recurring process of construction, transmission, preservation, and family mythmaking ${ }^{14}$.

Cette distinction de "patriciens», J. I. Little la relève également dans sa biographie consacrée à Henri-Gustave Joly de Lotbinière: "Quite conscious of his distinguished heritage, Joly was strongly attached to the seigneury that signified his patrician status ${ }^{15}$."

Notre enquête sur la mémoire seigneuriale vise justement à consigner ces pans d'une culture familiale issue de la transmission d'une conscience de soi propre aux anciennes familles seigneuriales. Elle ne concerne pas que les familles issues de la noblesse, elle prend pour objet le groupe des "derniers seigneurs », c'est-à-dire ceux qui détiennent des droits seigneuriaux au moment de l'ultime abolition (1940), lesquels peuvent appartenir à des strates diverses de la population, mais ont tous en commun une distinction sociale fondée sur la seigneurie ${ }^{16}$. C'est que, faut-il le rappeler,

11. Éric Mension-Rigau, Aristocrates et grands bourgeois. Éducation, traditions, valeurs (Paris, Perrin, 1997).

12. Éric Mension-Rigau, La vie des châteaux. Mise en valeur et exploitation des châteaux privés dans la France contemporaine. Stratégies d'adaptation et de reconversion (Paris, Perrin, 1999) et son plus récent ouvrage: Singulière noblesse. L'héritage nobiliaire dans la France contemporaine (Paris, Fayard, 2015).

13. Brian Young, "Revisiting Feudal Vestiges in Urban Quebec», dans Nancy Christie, dir., Transatlantic Subjects. Ideas, Institutions and Social Experience in Post-Revolutionnary British North America (Montréal et Kingston, McGill-Queen’s University Press, 2008), p. 133-156.

14. B. Young, Patrician Families..., p. 12.

15. J. I. Little, The Public and Private Life of Sir Henri-Gustave Joly de Lotbinière (Toronto, University of Toronto Press, 2013), p. 13.

16. Cette distinction, au sens où l'entend Bourdieu, revendiquée ou non par les membres du groupe seigneurial, participe plus largement de la reproduction sociale des élites et des modes de représentation propres à celles-ci. Pierre Bourdieu, La distinction, critique sociale du jugement (Paris, Les éditions de Minuit, 1979). 
tous les propriétaires seigneuriaux n’appartiennent pas nécessairement à la grande bourgeoisie ou à l'aristocratie et ne pourraient pas forcément revendiquer l'appartenance au patriciat étudié par B. Young ${ }^{17}$.

Michel Morissette a bien montré la diversité du groupe seigneurial après 1854, dans la continuité de la diversité qui le caractérisait déjà avant l'abolition $^{18}$. Ce groupe, bien que diversifié, partage néanmoins un statut distinctif fondé sur la possession de «droits» seigneuriaux (terres ou rentes). L'altérité seigneuriale ${ }^{19}$, tributaire du rapport d'autorité entre seigneurs et censitaires, constitue une forme de différenciation sociale classique des sociétés rurales anciennes ${ }^{20}$. Les modalités de l'abolition de la seigneurie au Québec auront permis à cette forme d'altérité de se perpétuer, par le biais notamment de la reproduction sociale des familles, dans le temps long jusque, à tout le moins, au milieu du $\mathrm{XX}^{\mathrm{e}}$ siècle comme nous le verrons. Notre étude vise, à partir du discours de certains de ces individus, à identifier ces habitus et ces pratiques qui fondent, à leurs yeux, la distinction de leur famille ${ }^{21}$. Cette mémoire seigneuriale est tantôt matérielle (documents, objets) ou visuelle (photographies, paysages, architecture), tantôt langagière ou sonore, voire généalogique. Elle s'exprime par la valeur accordée à cette filiation singulière et à la "mythologie» familiale qui s’y rattache. C'est ainsi que nous parlons de mémoire seigneuriale.

Cette recherche postule qu'à l'échelle locale, en particulier lorsque la famille seigneuriale a continué à résider sur place, la conscience de ce statut seigneurial - de ce rapport d'altérité - s'est maintenue bien après l'abolition, parfois jusqu'à très récemment. Toutefois, cette mémoire s'effrite et s'atténue au fil des générations. Les aînés qui la portent sont de précieux témoins et le temps nous est compté pour recueillir leurs souvenirs. La lecture de la nécrologie d'Edmond Joly de Lotbinière, "seul héritier d'une des plus anciennes seigneuries du Canada ${ }^{22}$ », au moment

17. B. Young, Patrician families...

18. Michel Morissette, "Les persistances de 1'“Ancien Régime” québécois: seigneurs et rentes seigneuriales après l'abolition (1854-1940)", mémoire de maîtrise (histoire), Université de Sherbrooke, 2014.

19. Le concept d'altérité en sciences sociales est d'usage commun. Toutefois nous avons abordé dans nos travaux cette notion d'altérité en contexte seigneurial pour mettre l'accent sur cette distinction sociale, souvent doublée d'une altérité ethnoculturelle, dans le monde rural laurentien. Voir par exemple: Benoît Grenier, Seigneurs campagnards de la nouvelle France. Présence seigneuriale et sociabilité rurale dans la vallée du Saint-Laurent à l'époque préindustrielle (Rennes, Presses universitaires de Rennes, 2007).

20. Voir entre autres: Jean Jacquart, Histoire de la France rurale, tome 2 de 1340 à 1789 (Paris, Seuil, 1975), p. 264 et 285 . Voir aussi Roland Mousnier, "Les communautés territoriales. Les seigneuries», dans Les institutions de la France sous la monarchie absolue 1598-1789. Tome I, Société et État (Paris, Presses universitaires de France, 1974), p. 371-427.

21. É. Mension-Rigau, Aristocrates et grands bourgeois..., p. 12-13.

22. Nécrologie d'Edmond Joly de Lotbinière, Le Soleil, 13 février 2014. 
même d'entreprendre cette recherche, en 2014, en était une regrettable confirmation. Après avoir esquissé les objectifs et le cadre méthodologique de cette recherche, nous dresserons un certain nombre de constats préliminaires pour définir l'identité familiale seigneuriale en formulant l'hypothèse d'une culture familiale distinctive, érigée sur une mémoire et une filiation enracinées dans l'histoire et dans la terre des anciennes seigneuries. Pour terminer, nous émettrons un certain nombre de pistes de réflexion quant aux valeurs découlant de cet héritage singulier.

\section{LA RECHERCHE SUR LA MÉMOIRE SEIGNEURIALE}

Il ne s'agit pas ici d'écrire l'histoire seigneuriale au $\mathrm{XX}^{\mathrm{e}}$ siècle, mais plutôt de colliger et d'interpréter la mémoire seigneuriale, telle que construite par les principaux intéressés au sein de récits familiaux. Il est trop tard, bien sûr, pour interroger ceux qui ont connu l'ultime phase de l'histoire du régime seigneurial. Dans un texte de février 1941, Gabrielle Roy, alors journaliste, faisait état de rencontres avec des censitaires. Elle citait même les propos de deux «vieux» habitants interviewés quelques mois plus tôt $t^{23}$. Sans pouvoir retrouver, comme Gabrielle Roy, des témoins qui auraient vécu personnellement les événements en question, il ne nous apparaissait cependant pas vain de mener une enquête orale sur cette mémoire singulière. En effet, lors de conférences prononcées à propos des survivances seigneuriales, des aînés m'ont relaté des souvenirs qui corroboraient les propos consignés par G. Roy. D’autres conservent une mémoire associée à leurs fonctions qui les ont mis en contact avec les réminiscences légales ou comptables de la propriété seigneuriale : notaires, secrétaires-trésoriers de municipalités et juges. Qui plus est, il est possible d'interroger les enfants et les petits-enfants de certains de ces derniers seigneurs, notamment pour connaître la manière dont on leur a transmis la mémoire de ces événements et de leur statut distinctif.

23. Gabrielle Roy, «Mort d'extrême vieillesse», Le Bulletin des Agriculteurs, 37, 2 (février 1941), p. 8 et 34-35. Nous avons consacré un article à la lecture que fait Gabrielle Roy de ces événements: Benoît Grenier, "Gabrielle Roy et le régime seigneurial québécois (1941)», Cahiers franco-canadiens de l'Ouest, 28, 2 (2016), p. 217-251. 


\section{La mémoire seigneuriale: un objet inexploré}

Homme de distinction, de générosité et de bonté, il fut le fils unique, seul héritier d'une des plus anciennes seigneuries du Canada. Concédée à René-Louis Chartier de Lotbinière en 1672 par Jean Talon, Intendant de Louis XIV, la seigneurie est restée dans la famille jusqu'en $1967^{24}$.

Comment, en lisant cette nécrologie d'Edmond Joly de Lotbinière, ne pas songer au sous-titre du livre de Georges Baillargeon, paru en 1968, Un régime qui ne veut pas mourir ${ }^{25}$ ? Voilà un exemple parmi tant d'autres de la survivance d'une forme de légitimité seigneuriale au-delà même du XXI siècle. C'est sur les traces de cette mémoire que nous nous sommes lancés. La mémoire seigneuriale, telle que nous la concevons, ne constitue pas, à ce jour, un objet d'étude au Québec, autant chez les historiens que chez les ethnologues ou même les historiens de l'art. Les littéraires, en s'intéressant de près au seigneur et auteur Philippe Aubert de Gaspé, sont parmi les seuls à avoir proposé une réflexion en ce sens ${ }^{26}$. Même dans une perspective purement patrimoniale, très rares sont les travaux qui portent un regard sur l'institution seigneuriale ${ }^{27}$. Cette lacune parait d'autant étonnante que la seigneurie a été la première institution d'encadrement de la population: "la seigneurie a précédé tout le reste ${ }^{28}$ », écrivait naguère Louise Dechêne. Implanté avant la paroisse, le régime seigneurial n’a pourtant pas donné lieu à la même prégnance dans l’imaginaire collectif québécois que les églises paroissiales et le patrimoine religieux dans son ensemble ${ }^{29}$. Récemment, quelques spécialistes se sont cependant penchés sur les processus de patrimonialisation, visant à saisir le travail mémoriel qui s'exerce autour des objets patrimoniaux, notamment des biens sei-

24. Nécrologie d'Edmond Joly de Lotbinière...

25. Georges-E. Baillargeon, La survivance du régime seigneurial à Montréal. Un régime qui ne veut pas mourir (Ottawa, Cercle du Livre de France, 1968).

26. Marc-André Bernier et Claude La Charité, dir., Philippe Aubert de Gaspé mémorialiste (Québec, Les Presses de l’Université Laval, 2009).

27. On peut évoquer quelques exceptions relatives au patrimoine bâti, dont le très ancien ouvrage de l'archiviste Pierre-Georges Roy, Vieilles maisons, vieux manoirs (Commission des monuments historiques de la province de Québec, 1927) ou l'inventaire de Raymonde Gauthier, publié en 1976 dans la foulée de l'adoption de la Loi sur les biens culturels par l'Assemblée nationale du Québec en 1972: Les manoirs du Québec (Montréal et Québec, Fides et éditeur officiel du Québec, 1976). Certains travaux d'érudits locaux et quelques études concernant les moulins ou relatives à un seul bâtiment pourraient aussi être cités.

28. Louise Dechêne, Habitants et marchands de Montréal au XVII siècle (Montréal, Boréal, 1988 [Plon, 1974]), p. 241.

29. Luc Noppen et Lucie K. Morisset, Les églises du Québec : un patrimoine à réinventer (Québec, Presses de l'Université du Québec, 2005). Laurier Turgeon et Louise Saint-Pierre, «Le patrimoine immatériel religieux au Québec: sauvegarder l’immatériel par le virtuel», Ethnologies, 31, 1 (2009), p. 201-233. 
gneuriaux $^{30}$. Jean-René Thuot et Maude Flamand-Hubert ont révélé, à travers l'exemple de L'Isle-Verte, que le patrimoine local et la mémoire seigneuriale peuvent être l'objet de mémoires concurrentes ou consécutives, invitant du même coup à « débusquer les mémoires » qui participent à nourrir les représentations du passés1.

\section{Mémoire de soi, mémoire de l'Autre: les enquêtes orales}

À l'origine, notre processus d'enquête orale visait une seule catégorie de seigneurs: les individus et familles (par opposition aux institutions). Nous interpellions les souvenirs relatifs aux rentes ou aux terres seigneuriales postérieures à l'abolition ${ }^{32}$. Or, en cours de route, nous avons jugé pertinent d'inclure la mémoire institutionnelle par l’intermédiaire du Séminaire de Québec et du Séminaire de Saint-Sulpice de Montréal ${ }^{33}$. Nous envisagions initialement d'identifier une dizaine de localités où la mémoire seigneuriale était plus susceptible d'être vivante, notamment en raison de la résidence sur place des "seigneurs», minimalement de façon saisonnière ${ }^{34}$. Nous avions pour ambition de rencontrer non seulement des familles d'ascendance franco-catholique, mais aussi des familles angloprotestantes pour refléter l'importante part des anglophones au sein du groupe des seigneurs ${ }^{35}$. Qui plus est, à l'instar des Joly de Lotbinière, certaines de ces familles ont été particulièrement enclines à des alliances

30. Par exemple: Étienne Berthold, Patrimoine, culture et récit : l'île d'Orléans et la Place Royale de Québec (Québec, Les Presses de l’Université Laval, 2012).

31. Maude Flamand-Hubert, «La maison Louis-Bertrand: lieu de mémoire et d'histoire», dans Karine Hébert et Julien Goyette, dir., Histoire et idées du patrimoine, entre régionalisation et mondialisation (Québec, éditions MultiMondes, 2010), p. 141-160; Jean-René Thuot, «L'imaginaire seigneurial: les points de convergence entre recherche fondamentale, initiatives touristiques et mémoires communautaires ", dans B. Grenier et M. Morissette, dir., Nouveaux regards..., p. 364-397 et Mathieu Lévesque-Dupéré, «Vieux manoirs vieilles maisons : la patrimonialisation des résidences seigneuriales sur la Côte du Sud», mémoire de maîtrise (histoire), Université de Sherbrooke, 2018.

32. Cette précision était importante puisqu'il ne s'agissait pas de recueillir la mémoire de toutes les familles seigneuriales, mais bien de celles en place au moment de l'extinction du régime (1854-1940). Il a d'ailleurs été nécessaire de décliner, poliment, les propositions de certains volontaires qui se présentaient comme des descendants de tel ou tel seigneur de l'époque de la Nouvelle-France ou dont la famille s'était départie de ses propriétés avant l'abolition.

33. Il s'est avéré que la mémoire institutionnelle de la seigneurie, telle que construite et transmise au sein de ces institutions, représentait un objet d'étude non moins intéressant que la mémoire familiale. Nous avons consacré un article à cette question: Benoît Grenier, «Le patrimoine seigneurial du Séminaire de Québec ou l'héritage matériel de François de Laval», dans Étienne Berthold, dir., Le patrimoine des communautés religieuses. Empreintes et approches (Québec, Les Presses de l'Université Laval, 2018), p. 21-51.

34. Le critère de la "présence seigneuriale» était au centre de notre recherche doctorale et demeure, à nos yeux, un signe additionnel de distinction des familles seigneuriales: B. Grenier, Seigneurs campagnards...

35. Au milieu du XIX ${ }^{e}$ siècle, les anglophones comptent pour au moins la moitié des propriétaires seigneuriaux du Québec. Fernand Ouellet, «Propriété seigneuriale et groupes sociaux dans la vallée du Saint-Laurent (1663-1840)", Revue de l'Université d'Ottawa, 47,1-2 (1977), p. 183-213. Voir aussi: Alex Tremblay- 
mixtes aux $\mathrm{XIX}^{\mathrm{e}}$ et $\mathrm{XX}^{\mathrm{e}}$ siècles. Il s'agissait d'être sensibles à l'impact des facteurs de l'ethnicité et de la religion comme indicateurs d'une altérité additionnelle au rapport seigneur/censitaire dans les campagnes seigneuriales. Dans la mesure du possible, nous escomptions aussi rencontrer les aînés de ces mêmes localités, porteurs de la mémoire locale ce qui fut, on le verra, plus difficile à réaliser.

L'enquête sur la mémoire seigneuriale ne visait pas à approfondir la connaissance sur les acteurs interrogés et leur vécu, mais à saisir une perception, une représentation, de ces survivances à l'échelle locale; les faits, quant à eux, sont déjà bien documentés par les sources écrites ${ }^{36}$. Comme l'affirme Hélène Wallenborn: "La source orale, même quand elle est factuellement erronée, est "vraie" du point de vue du narrateur. Elle parle moins des événements que des significations qu'il leur donne ${ }^{37}$." Les individus qui portent cette mémoire sont souvent âgés. Toutefois, comme le soulignait Denyse Baillargeon, la mémoire «des personnes âgées, lorsqu'elles sont en bonne santé, n'est pas moins fiable que celle d'un jeune adulte et $[\ldots]$ les pertes de mémoire reliées au vieillissement affectent en premier lieu la mémoire récente, laissant intacts les souvenirs d'enfance et de jeunesse ${ }^{38} »$. Ce sont ces souvenirs, anciens, et même les souvenirs de famille transmis par leurs parents, qui nous intéressaient tout particulièrement ${ }^{39}$. Au terme de l'exercice, nous avons la conviction que l'histoire orale, aussi étonnant que cela puisse paraître, aura contribué à mieux saisir une dimension de l'histoire nationale - le régime seigneurialqui est trop souvent confinée à la période préindustrielle.

Lamarche, "La stabilisation et la créolisation de la présence seigneuriale britannique dans la vallée du Saint-Laurent, 1790-1815 ", dans B. Grenier et M. Morissette, dir, Nouveaux regards ..., p. 247-276.

36. Dans son étude sur la mémoire collective des Acadiens, telle que colligée lors des célébrations de leur $400^{\mathrm{e}}$ anniversaire, Ronald Rudin s'est penché sur les représentations des diverses communautés (Acadiens, anglophones des Maritimes, Premières Nations) et du sens à donner à ces événements: Ronald Rudin, L'Acadie entre le souvenir et l'oubli. Un historien sur les chemins de la mémoire collective (Montréal, Boréal, 2014). Notre étude s'inspire en quelque sorte de son approche, mais à l'échelle de la mémoire «familiale» d'un groupe spécifique. Qui plus est, comme Rudin, nous avons fait usage du documentaire comme médium de diffusion de nos travaux.

37. Hélène Wallenborm, L'historien, la parole des gens et l'écriture de l'histoire: le témoignage à l'aube du $X X I^{e}$ siècle (Loverval, éditions Labor, 2006), p. 50-51.

38. Denyse Baillargeon, Ménagères au temps de la Crise (Montréal, Les éditions du remue-ménage, 1993), p. 32.

39. Pour une réflexion approfondie sur la source orale et l'historique de son usage chez les historiens du Québec, nous renvoyons le lecteur au numéro thématique de la Revue d'histoire de l'Amérique française, 69, 1-2 (été-automne 2015), ainsi qu’à la contribution de Steven High et Jean-Philippe Warren, «Memory of a Bygone Era: Oral History in Quebec, 1979-1986», The Canadian Historical Review, 95, 3, (2014), p. 433-456. Voir également le texte fondateur de Paul Thompson, «Des récits de vie à l’analyse du changement social», Cahiers internationaux de sociologie, 69 (1980), p. 249-268. 
Nous $^{40}$ avons bénéficié du soutien de plusieurs partenaires qui ont contribué à retracer ces témoins, somme toute peu nombreux. Le Musée de la mémoire vivante, érigé à Saint-Jean-Port-Joli dans la reconstruction du manoir de Philippe Aubert de Gaspé, s'est révélé un précieux partenaire et les témoignages recueillis dans le cadre de notre enquête y ont été déposés en juin $2018^{41}$, avec le consentement des participants, à des fins de conservation et de diffusion ${ }^{42}$. Une exposition portant sur l'histoire et la mémoire seigneuriale du Québec devrait voir le jour à la suite de cette collaboration.

Entre février 2015 et décembre 2017, nous avons réalisé 34 entretiens et parcouru plus de 15000 kilomètres dans le territoire «seigneurial» du Québec, nous conduisant dans 16 anciennes seigneuries, du Bic à Vaudreuil et de Saint-Georges-de-Beauce à Terrebonne en passant par la région de Portneuf. Les témoins ( 20 hommes et 14 femmes), dont l'âge varie entre 8 et 95 ans, nous ont permis de rencontrer une dizaine de familles dont certaines mieux représentées que d'autres. Ainsi, chez les LotbinièreHarwood, nous avons pu croiser les interprétations de trois générations (six individus) de la même famille; dans plusieurs autres cas, nous avons pu bénéficier de celles de deux générations ou encore de fratries, comme dans le cas des Pozer en Beauce. Cette lecture générationnelle est particulièrement intéressante pour saisir les différentes perceptions d'une même histoire familiale, lesquelles sont nécessairement influencées par l'âge des témoins, mais aussi par l'éloignement plus ou moins grand des événements racontés. Malgré cet écart d'âge en apparence très considérable (87 ans entre l'aînée et la cadette), l'âge moyen des témoins rencontré est de 62,6 ans $(60,1$ ans pour les femmes et 64,3 ans pour les hommes).

De toute évidence, le premier biais dans l'échantillonnage réside dans la nature volontaire de l'exercice. Les individus qui, spontanément, se sont manifestés pour témoigner de leur «histoire » seigneuriale avaient nécessairement conscience de ce bagage. Ceux à qui rien n’a été transmis ou

40. Le nous dans ce texte est un véritable nous "collectif». Toute cette enquête a été le fruit d'un important travail d'équipe entre Stéphanie Lanthier à la caméra, un assistant de recherche - collaborateur à la technique - et moi-même.

41. Musée de la mémoire vivante, <www.memoirevivante.org/>.

42. D’autres partenaires méritent d'être mentionnés, notamment la Fédération Histoire Québec qui a contribué à la diffusion de l'appel auprès des diverses sociétés d'histoire, le Musée régional de VaudreuilSoulanges et les Seigneuriales de Vaudreuil-Dorion, la Corporation de la Seigneurie des Aulnaies, la Corporation des Moulins de l'ssle-aux-Coudres, la Société régionale d'histoire de Terrebonne et la Société d'art et d'histoire de Beauport. Une page Facebook consacrée à la recherche a aussi permis de contribuer à la visibilité du projet et à susciter la participation de certains témoins. 
pour qui cet héritage n'a pas ou peu d'importance risquent fort de ne pas s'être sentis interpellés. Toutefois, certains ont été "convaincus» par des membres de leur entourage même s'ils y voyaient initialement peu d'intérêt. Un témoin potentiel, dont plusieurs personnes m'avaient parlé, a, au contraire, malgré une identité seigneuriale «forte», préféré ne pas se livrer publiquement, considérant que «c'est de la famille et non de l'histoire» et faisant valoir le caractère privé de sa propriété. Cependant, la grande majorité des individus qui ont appris l'existence de la recherche ont été très généreux de leur temps et de leurs souvenirs. Il faut donc avoir conscience que la mémoire seigneuriale ici interprétée est, de toute évidence, celle d'individus pour qui la seigneurie et l'histoire familiale ont une importance parce qu'elle leur a été transmise. Gardons-nous donc d'interpréter pour l'ensemble du groupe des descendants de seigneurs, dont plusieurs ne résident plus au Québec depuis longtemps ou n'ont tout simplement pas la connaissance de ce passé distinctif. Toutefois, comme nous le verrons plus loin, les individus rencontrés, dont la plupart ne se connaissent pas, partagent de nombreuses caractéristiques communes ${ }^{43}$.

Une deuxième limite qu'il convient de mentionner concerne la difficulté à recruter des "aînés" porteurs du souvenir des rentes seigneuriales, ce que nous appelions la «mémoire de l'Autre». En effet, l'appel invitait toute personne qui avait des souvenirs relatifs aux rentes ou aux terres seigneuriales. Or, bien que de nombreux témoignages nous aient été livrés spontanément lors des périodes de questions à l’occasion de conférences, il ne semble pas que ces micro-souvenirs leur aient paru suffisamment importants pour être partagés dans le cadre d'une recherche ${ }^{44}$. On peut supposer que certains ont été intimidés par la nature "scientifique » et universitaire de l'enquête ou encore qu'ils sous-estimaient la valeur de leurs souvenirs. Cette dernière hypothèse est renforcée par le fait que même parmi les descendants de familles seigneuriales, dont certains avaient d'eux-mêmes pris contact avec moi, quelques-uns se sont finalement désistés en jugeant qu'ils ne voyaient pas ce qu'ils pouvaient avoir d'intéressant à dire. Dans

43. Comme c'est habituellement le cas en histoire orale, nous avons bien perçu, à un certain stade, l'atteinte du "point de saturation" au-delà duquel il devenait assez évident que de nouvelles entrevues ne feraient que confirmer les renseignements déjà recueillis: D. Baillargeon, Ménagères au temps de la Crise..., p. 37.

44. Je pense, par exemple, à cet homme âgé de 80 ans environ qui m’interpelle après une conférence à Sorel, en 2014, pour me relater un souvenir qui lui semblait tout frais en mémoire: "La seule fois où je me rappelle avoir vu ma grand-mère, toujours douce et aimable, se mettre en colère, c'est lorsque le collecteur des rentes seigneuriales venait à la maison. » Lors d'une conférence en mars 2015 au Musée de la mémoire vivante, nous avons recueilli quelques anecdotes de personnes présentes et désireuses de partager leurs souvenirs. 
ces cas, il s'agissait de les rassurer sur la valeur de leur témoignage sans pour autant avoir l'air de leur forcer la main ${ }^{45} \ldots$ Cette attitude, tout de même assez rare, a été constatée plus souvent chez des familles plus «modestes» ou roturières, pour recourir à un terme propre à l'Ancien Régime. La mémoire seigneuriale semble effectivement plus vive chez les familles d'ascendance nobiliaire ou bourgeoise.

Parmi les 34 témoins, on ne compte pas que des membres de familles seigneuriales. Ceux-ci se déclinent en 3 catégories qui se sont, au final, constituées d'elles-mêmes et sont devenues, au fil du temps, les 3 axes de la recherche sur la mémoire seigneuriale: la mémoire familiale (20 témoins), la mémoire institutionnelle ( 3 témoins) et les "porteurs» de mémoire ou témoins indirects des persistances seigneuriales (11 témoins). Nous avons analysé ailleurs la mémoire institutionnelle du Séminaire de Québec ${ }^{46}$. Quant aux porteurs de mémoire (meuniers, prêtres, notaires, personnes âgées des localités ou encore actuels propriétaires des biens seigneuriaux), ils feront l'objet d'un article séparé, compte tenu de la grande richesse de leurs témoignages qui se révèlent souvent complémentaires à ceux des familles et parfois même la seule manière d'accéder à la mémoire de familles qui se sont éteintes. Nous nous concentrerons dans les prochaines pages sur la mémoire familiale, telle qu'elle peut s'observer à partir des 20 témoignages recueillis auprès des membres de 8 familles distinctes, 5 francophones et 3 anglophones (ou mixtes). Le hasard a voulu que cet échantillonnage soit tout à fait représentatif de la réalité des familles seigneuriales du Québec, avec des descendants de familles francophones et anglophones, nobles (3) et roturières (5), certaines plus modestes que d'autres, mais également une représentativité sur le plan géographique.

Les entretiens réalisés consistaient en une entrevue semi-dirigée d'une durée moyenne de 60 à 90 minutes pendant laquelle les témoins étaient invités à aborder trois aspects: 1 . leurs origines familiales/seigneuriales (incluant les origines de la famille, les seigneuries possédées et ce qu'il en restera après l'abolition - du moins ce qu'ils en savent); 2 l'identité familiale et la transmission des valeurs associées; 3. l'héritage seigneurial (notamment sur le plan patrimonial). Compte tenu de l'objet d'étude, nous n’avons pas opté pour l'approche du «récit de vie», bien que plu-

45. Nous avons été à même de constater la nécessaire empathie dont doit faire preuve l'intervieweur, en particulier face aux personnes âgées. Sur ces questions: Sophie Chaxel, Cécile Fiorelli et Pascale Moity-Maïzi, «Les récits de vie: outils pour la compréhension et catalyseurs pour l'action », Interrogations, 17 (janvier 2014) [en ligne], <www.revue-interrogations.org/Les-recits-de-vie-outils-pour-la> .

46. B. Grenier, «Le patrimoine seigneurial du Séminaire de Québec...». 
sieurs des entretiens nous aient conduit à aborder différentes étapes (personnelles ou professionnelles) de la vie des témoins. Ceux-ci avaient le plus souvent été rencontrés préalablement ou lors d'un entretien téléphonique, ce qui a permis de les rassurer quant aux attentes: nul besoin de connaître l'histoire sur le bout des doigts. Le but de l'entretien était de comprendre leur vision des choses, leurs souvenirs, en somme la mémoire de la famille telle que transmise.

Nous avons pris la décision, dès les commencements, de filmer les entretiens en format HD. La collaboration de la cinéaste et historienne Stéphanie Lanthier, déjà aguerrie à l'histoire orale ${ }^{47}$, a été déterminante à cet égard. Non seulement souhaitions-nous consigner les témoignages, mais il s'agissait aussi de capter sur caméra ces "visages seigneuriaux», leur gestuelle, leurs silences, leur langage corporel, l'émotion, mais aussi l'agir du rappel mémoriel. Contrairement à ce que nous aurions pu redouter, la quasi-totalité a accepté très généreusement, même s'il s'agissait pour la plupart d’une première expérience devant la caméra, notamment pour les plus âgés. Le recours à la vidéo nous a d'ailleurs permis de filmer les lieux et les paysages associés à ces souvenirs. C'est que, dans la mesure du possible, nous avons voulu filmer les entretiens dans des lieux emblématiques de l'histoire des familles rencontrées, de manière à nourrir la mémoire des individus en leur offrant un environnement familier et évocateur. Plusieurs des entretiens ont été réalisés directement chez les témoins, surtout lorsqu'ils étaient toujours résidants, ou résidants saisonniers, de la seigneurie familiale. Ainsi, bien que la mémoire soit un processus «sélectif et conditionné par le pré$\operatorname{sent}^{48}$ », les souvenirs sollicités ont pu être ravivés par un tel «retour aux sources». Qui plus est, en renonçant à l'anonymat (la possibilité leur était tout de même offerte dans le formulaire de consentement), ils avaient le sentiment de contribuer à la pérennité de l'histoire de leur famille ou de leur localité. Enfin, nous avons souvent été confrontés à un curieux dilemme lors de nos rencontres avec ces familles. Bien que nous ayons été sur place pour réaliser une enquête orale et faire appel à leurs souvenirs, ce sont souvent leurs archives que ces familles tenaient à nous présenter (voire à nous confier!) lorsque nous débarquions chez eux. De véritables trésors documentaires se sont présentés à nous, mais il fallait trouver un juste milieu pour ne pas sembler désintéressés tout en gardant le cap sur l'objectif principal: la mémoire. Ce fut particulièrement remarquable chez les Rioux,

47. Stéphanie Lanthier a collaboré étroitement à l’enquête menée par Louise Bienvenue : «Mémoires de Boscoville», Espace CIEQ, < https: / / boscoville.cieq.ca/> ainsi qu’à différents autres projets depuis.

48. D. Baillargeon, Ménagères au temps de la Crise..., p. 32. 


\section{Figure I}

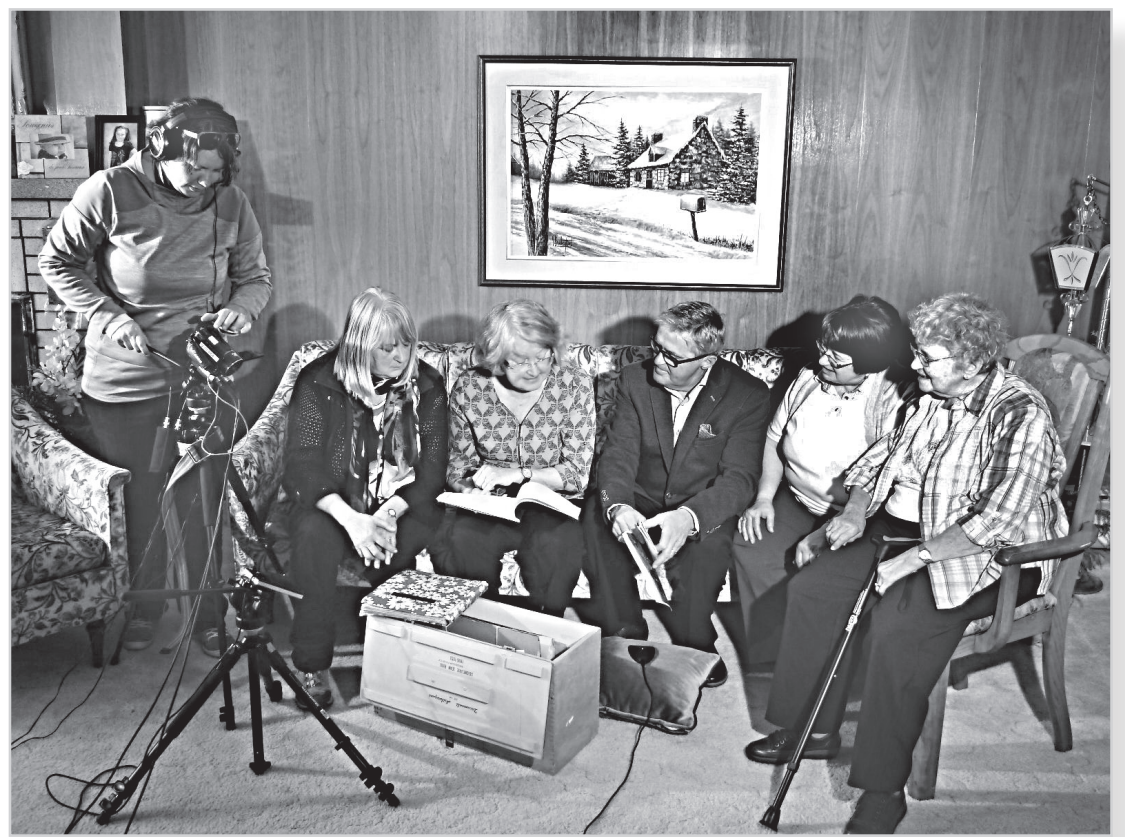

La famille Rioux sous la caméra de Stéphanie Lanthier,Trois-Pistoles, mai 2015

Crédit: Benoît Grenier

enracinés à Trois-Pistoles depuis 1697, où nous attendait un coffre de menuisier rempli des documents originaux relatifs à la seigneurie dont les plus anciens dataient du XVII siècle et les plus récents témoignaient de la gestion seigneuriale de la famille après l'abolition. Pour plusieurs, ces rencontres ont été non seulement une occasion de mettre de l'avant leur histoire familiale, avec une modestie variable, mais aussi bien souvent le prétexte à une véritable rencontre de famille. Nous avons été les témoins privilégiés de moments très touchants, à Trois-Pistoles, à Saint-Georges-de-Beauce, à Vaudreuil, au Bic... Nul besoin de dire que dans une telle enquête, l'objectivité de l'historien, habitué aux documents des XVII ${ }^{e}$ et XVIII ${ }^{e}$ siècles et aux acteurs historiques morts depuis belle lurette, est mise à mal ${ }^{49}$.

49. Le rapport entre l'interviewé et l'intervieweur est au cœur des enjeux éthiques propres à l'histoire orale, tout comme la confiance qui s'établit entre ces deux acteurs de la création de la source orale. Pour une réflexion sur l'archive orale: D. Baillargeon, Ménagères au temps de la Crise..., p. 35-36. Voir aussi: 
Outre les archives familiales, ce sont également les traces d'une culture matérielle seigneuriale que nous avons croisées durant cette enquête. Afin de stimuler la mémoire et les anecdotes, nous avions demandé, préalablement, à tous les témoins, d'avoir en main un objet qui puisse être significatif de leur histoire seigneuriale. Plusieurs ont sorti photographies anciennes, documents ou livres de la bibliothèque familiale. Dans certains cas, on nous a présenté de véritables trésors, comme ce petit coffret offert par LouisJoseph Papineau à sa petite-nièce Henriette Dessaules ${ }^{50}$. Toutefois, l'objet le plus remarquable, dont trois familles nous ont parlé mais qu'une seule possédait toujours, était la canne à pommeau d'or, dite «du seigneur». Chez Anita Rioux, 95 ans, on conserve en effet jalousement ce bel objet d'orfèvrerie réalisé en 1896 pour son père Joseph-Magloire Rioux, alors «seigneur» de Trois-Pistoles. Cet objet, qui n’est pas en soi distinctif de l'identité seigneuriale, participe de la culture matérielle masculine de l'âge victorien plus généralement ${ }^{51}$. Mais, à la différence d'autres grands bourgeois, souvent urbains, qui arboraient pareilles cannes au tournant du siècle, l'objet revêt, pour la famille Rioux, une dimension «seigneuriale » et lorsque J.-M. Rioux déambulait avec cet objet dans la petite municipalité de Trois-Pistoles, tout porte à croire que l'autorité seigneuriale était clairement manifestée, même quarante ans après l'abolition. D’autres objets pourraient aussi être révélateurs de cette culture matérielle seigneuriale, dont l'épée, illustration s'il en est une de l'autorité et de la masculinités2.

Steven High, «Au-delà de la "citation payante": les archives vivantes et la recherche réciproque en histoire orale", Revue d'histoire de l'Amérique française, 69, 1-2 (été-automne 2015), p. 137-163.

50. Entretien 2015-01 avec Marie-José Raymond, Saint-Paul d'Abbotsford, 13 février 2015.

51. Voir le travail de l'historienne Ariel Beaujot pour d'autres exemples de cette culture matérielle victorienne: Victorian Fashion Accessories (Londres et New York, Bloomsbury, 2012), et " "If you want to get ahead, get a Hat": Manliness, Power, and Politics via the Top Hat", Revue de la Société historique du Canada, 25, 2 (2014), p. 57-88.

52. L'épée pourrait effectivement constituer une autre marque distinctive de ces élites seigneuriales, dont plusieurs détiennent aussi fréquemment des fonctions de commandement dans l'armée. Nous n'avons cependant pas eu d'échos de telles possessions chez les témoins rencontrés. Il est fort probable qu'il en aurait été autrement au tournant du $\mathrm{XX}^{\mathrm{e}}$ siècle comme porte à le croire la biographie de De Saint-Denys Garneau par Michel Biron: De Saint-Denys Garneau. Biographie (Montréal, Boréal, 2015), notamment le chapitre premier intitulé «Le manoir». La récente thèse de Virginie Chaleur-Launay insiste, sans toutefois aborder longuement la culture matérielle, sur la dimension militaire de cette famille de la noblesse seigneuriale après la Conquête: Virginie Chaleur-Launay, «Les Salaberry entre deux empires. L'adaptation d'une famille de la noblesse canadienne-française sous le régime anglais», thèse de doctorat (histoire), Sorbonne-Université, 2019. 


\section{Figure 2}

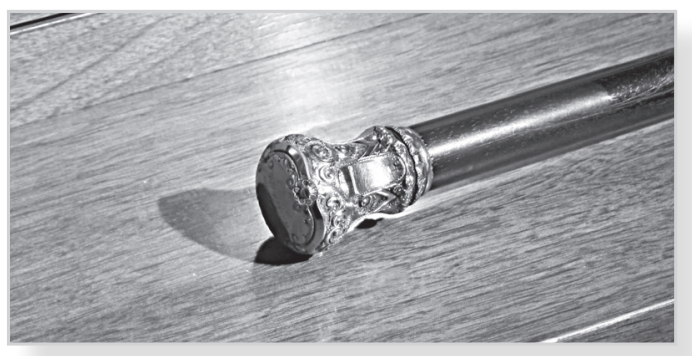

Canne du seigneur Joseph-Magloire Rioux (1896),

Trois-Pistoles Crédit: Benoît Grenier

\section{UNE CULTURE FAMILIALE SEIGNEURIALE?}

\section{L'oubli des rentes seigneuriales... et l'omniprésence de la terre}

Avant d'évoquer ce qui ressort des entretiens avec les descendants des derniers seigneurs québécois, l'honnêteté intellectuelle oblige à dire ce qui fut pratiquement absent des discussions, malgré nos questions à ce sujet. Ayant déjà étudié attentivement la propriété des rentes constituées et la nature des indemnités reçues par les propriétaires de ces rentes au terme de la ligne du temps seigneuriale, j'imaginais retrouver la mémoire de ces redevances chez les témoins rencontrés. Or, très rares furent les allusions aux rentes seigneuriales qui se sont pourtant maintenues entre 1854 et 1940. Ma recherche arrive visiblement trop tard; il eut fallu que Marcel Trudel se lance, dès les années 1950 , sur la trace des vestiges seigneuriaux ${ }^{53} \ldots$ Vu l'âge moyen des participants, il n'est guère surprenant qu'ils n'aient pas de souvenirs relatifs à ces questions très pragmatiques liées aux rentes seigneuriales possédées par leurs aïeux. Déception? Pas réellement, puisque nous savons, par les sources écrites produites par le Syndicat national du rachat des rentes seigneuriales ${ }^{54}$, que les parents ou grands-parents de ces personnes ont possédé ces droits ${ }^{55}$. Cependant, force est de constater que cela ne semble pas constituer un élément mémoriel significatif. Anita Riou(x) (1920-2017) est l'exception à la règle. Âgée de 95 ans au moment de l'entretien et fille unique du seigneur Joseph-Magloire Riou, elle est la seule à se souvenir personnelle-

53. Marcel Trudel en est d'ailleurs pleinement conscient lorsqu'il rédige sa fameuse brochure en 1956, puisqu'il consacre les dernières lignes à cette question des rentes seigneuriales, qui venaient alors de prendre fin: Marcel Trudel, Le régime seigneurial (Ottawa, Société historique du Canada, 1956). p. 19.

54. BAnQ-Québec, Fonds E39, Syndicat national du rachat des rentes seigneuriales.

55. Benoît Grenier (avec la collaboration de Michel Morissette), «Les persistances de la propriété seigneuriale au Québec. Les conséquences d'une abolition partielle et progressive (1854-1970)", Histoire et Sociétés Rurales, 40, 2 (2013), p. 61-96 et B. Grenier, «Le dernier endroit dans l'univers...». 
ment du paiement des rentes à la maison en novembre de chaque année jusqu'en 1940. Toutefois, son témoignage confond les rentes constituées, qui s'arrêtent en 1940, et les rentes que la famille a récoltées jusqu'au début du XXI ${ }^{e}$ siècle pour des terres tenues en loyers par des résidents de TroisPistoles $^{56}$. Les archives et notre connaissance des faits nous permettent cependant de démêler les fils de ce précieux témoignage.

Par contre, le second aspect de la propriété seigneuriale post-abolition, soit les propriétés foncières détenues par ces familles après 1854 (et parfois jusqu'à nos jours), a laissé une trace bien plus considérable chez les familles rencontrées. Il en va de l'ancrage au sol - à la terre - une dimension fortement chargée sur le plan identitaire et affectif, comme le soutient Éric Mension-Rigau à propos des familles nobles françaises: "[Sans la terre], l'identité noble est orpheline, [elle] maintient un point d'ancrage dont l'existence aide à la transmission de l'identité nobiliaire ${ }^{57}$ ». Cependant, à l'encontre des châtelains français, peu de familles rencontrées demeurent toujours dans la résidence ancestrale, le manoir a souvent disparu ou encore la famille s'en est départie au fil du temps, non sans douleur comme l'ont attesté plusieurs témoins; autant de témoignages de notre difficile rapport comme société au patrimoine bâti. Selon le Registre du patrimoine culturel du Québec, un seul manoir seigneurial reste la propriété de la famille: «le domaine seigneurial appartient toujours aux descendants des seigneurs de Saint-Ours, ce qui constitue un cas unique au Québec ${ }^{58}$ ». Malheureusement, le propriétaire des lieux, décédé récemment, n’a pu répondre à nos questions.

Pour les familles qui résident encore sur leur ancien fief, comme les Rioux à Trois-Pistoles, cette dimension est particulièrement forte. D'autres n'y ont conservé qu'une résidence secondaire, par exemple la famille de Douglas Cann, dont les terres familiales ont été expropriées en partie pour la création du Parc national du Bic au début des années 1970. Sa sœur et lui possèdent néanmoins toujours chacun une propriété dans la seigneurie dont leur aïeul Archibald Campbell a été le premier seigneur à résider sur place, de manière saisonnière. Ils y passent tous leurs étés même s'ils résident respectivement à Chicago et à London (Ontario). L'attachement

56. Entretien 2015-03 avec Anita Rioux, Trois-Pistoles, 15 mai 2015.

57. É. Mension-Rigau, La vie des châteaux..., p. 337.

58. "Domaine seigneurial de Saint-Ours», Registre du patrimoine culturel du Québec, en ligne : <www. patrimoine-culturel.gouv.qc.ca $/ \mathrm{rpcq} /$ detail.do ? methode $=$ consulter\&id $=92494 \&$ type $=$ bien $\#$. VzNwp-R0pjg>. 
au sol, à la nature (notamment aux cours d'eau) et aux paysages est viscéral. Douglas Cann déclare en entrevue que son cœur est au $\mathrm{Bic}^{59}$.

Plus largement, cela fait écho à une identification aux lieux, indépendamment du maintien d'une résidence sur place. «Je me sens ici chez moi», nous a confié M.-J. Raymond à propos de la région de Saint-Hyacinthe ${ }^{60}$. Plusieurs témoins l'ont formulé d'une manière assez similaire, révélant un attachement très fort à cette terre de leur famille. L'un d'eux nous a même confié que pour son père, aujourd'hui décédé, ces terres seigneuriales représentaient une véritable obsession à la fin de sa vie. Ce sentiment d'appartenance, on peut le ressentir dans l'œuvre et les écrits privés de la romancière Anne Hébert, née à Sainte-Catherine-de-Fossambault, localité dont ses ancêtres étaient seigneurs ${ }^{61}$. Dans un texte paru en 1972, elle écrivait: «Ma plus profonde terre, en ce monde, c'est sans doute Sainte-Catherine, avec sa rivière et son paysage sombre et morcelé et puis Québec, ouvert sur la beauté du fleuve, puis replié sur lui-même, dans le secret de ses maisons fermées et de ses vieilles familles ${ }^{62}$.»

Ce sentiment d'être "chez soi» peut prendre différentes formes, mais au tournant du XXe siècle et certainement jusqu'aux années 1950 (selon les témoignages et les sources écrites), les familles seigneuriales, même sur leur déclin, agissent encore comme au temps de leurs aïeux. C’est le cas pour Hermine Prévost (1885-1953), mère du poète De Saint-Denys Garneau (1912-1943), à Sainte-Catherine-de-Fossambault. Dans sa biographie du poète, Michel Biron écrit à son sujet: «Hermine se sent partout chez elle à Sainte-Catherine, comme si tout le village constituait encore symboliquement une propriété familiale ${ }^{63}$.» Elle se comportait en «seigneuresse », ajoute à son sujet Odette Dick, actuelle propriétaire du manoir seigneurial de l'endroit ${ }^{64}$. Ces seigneurs, souvent résidants saisonniers, visitent leurs "censitaires», lorsqu'ils arrivent sur place annuellement. Douglas Cann, âgé de 65 ans, se rappelle de sa mère, Grace Cecily Margaret Gibbs, qui agissait comme «la grande dame qui fait ses visites»,

59. Entretien 2015-10 avec Douglas Cann, Le Bic, 7 août 2015.

60. Entretien 2015-01 avec Marie-José Raymond, Saint-Paul d'Abbotsford, 13 février 2015.

61. Benoît Grenier, «L'héritage seigneurial d'Anne Hébert: famille et enracinement comme marqueurs identitaires", Les Cahiers Anne Hébert, 15 (2018), p. 7-29.

62. Anne Hébert, "Les étés de Kamouraska ... et les hivers de Québec», Le Devoir, octobre 1972, publié dans Anne Hébert, Euvres complètes d'Anne Hébert, volume V Théâtre, nouvelles et proses diverses, éditions établies par Patricia Godbout, Annie Tanguay et Nathalie Watteyne (Montréal, Presses de l’Université de Montréal, 2015), p. 924.

63. M. Biron, De Saint-Denys Garneau..., p. 23.

64. Entretien 2015-13 avec Odette Deschênes-Dick, Sainte-Catherine-de-la-Jacques-Cartier, 13 novembre 2015 . 
entrant sans frapper chez les "paysans» du $\mathrm{Bic}^{65}$. Ces comportements relèvent à la fois d'une familiarité avec les habitants, certainement d'un attachement à leur égard, tout autant que d'une forme de paternalisme caractéristique du rapport d'altérité propre au régime seigneurial que n’aurait pas reniée Philippe Aubert de Gaspé.

\section{LE MAINTIEN D'UN RAPPORT D'ALTÉRITÉ DANS LES CAMPAGNES POST-SEIGNEURIALES}

Le mode de vie seigneurial ne s'éteint pas, loin s'en faut, avec l'abolition. La construction de nombreux manoirs seigneuriaux, à l'époque de l'abolition et après celle-ci, rappelle bien que les familles seigneuriales continuent à jouir d'une autorité symbolique sur les populations de leurs fiefs. La Seigneurie des Aulnaies, centre d'interprétation du régime seigneurial, est une excellente illustration de cette réalité. Le manoir, construit pour Amable Dionne en 1853, un an avant l'abolition, demeurera jusqu'au tournant du $\mathrm{XX}^{\mathrm{e}}$ siècle un haut lieu de la sociabilité des élites régionales, comme le souligne la visite guidée du manoir ${ }^{66}$. L'élection de plusieurs «anciens » seigneurs à la mairie des municipalités qui émergent alors n'est qu'une des illustrations du maintien de cette autorité locale ${ }^{67}$. Cette altérité se manifeste de manière bien concrète dans les communautés rurales et sous plusieurs formes, notamment par les titres ou autres marques de déférence, mais aussi par le maintien des «droits honorifiques » à l'église, comme le banc seigneurial et l'inhumation sous celui-ci, envers les membres de la première famille des lieux.

L'exemple le plus révélateur est certainement celui d'Anita Rioux, longtemps surnommée la «seigneuresse » à Trois-Pistoles. Issue d'une famille seigneuriale roturière pionnière, enracinée sur place depuis la fin du XVII

65. Entretien 2015-10 avec Douglas Cann, Le Bic, 7 août 2015.

66. Le site Web présente pourtant le site comme un "centre d'interprétation de la vie seigneuriale en Nouvelle-France [qui] contribue à vous faire découvrir plus de 350 ans d'histoire ", La seigneurie des Aulnaies: <www.laseigneuriedesaulnaies.qc.ca/>. Cela illustre à quel point la mémoire du régime seigneurial est ancrée dans un passé lointain, essentiellement associé au régime français malgré sa longévité exceptionnelle. Voir notre réflexion sur ce paradoxe: Benoît Grenier, «Les paradoxes de la mémoire seigneuriale au Québec: entre la mythologie et l'oubli», dans Marc Bergère et al., dir., Mémoires canadiennes (Rennes, Presses universitaires de Rennes, 2018), p. 155-166.

67. Le cas de la famille Fraser, à Rivière-du-Loup (Fraserville) en est une illustration parmi d'autres : B. Grenier et M. Morissette, "Propriétés et propriétaires seigneuriaux...». Voir aussi: Benoît Grenier, «Élites seigneuriales, élites municipales: Le pouvoir seigneurial à l'heure de l'abolition», dans Thierry Nootens et Jean-René Thuot, dir., Les figures du pouvoir à travers le temps: Formes, pratiques et intérêts des groupes élitaires au Québec, XVII'-XX'e siècles (Québec, Les Presses de l’Université Laval, 2012), p. 57-64. 
siècle $^{68}$, Anita Riou ${ }^{69}$ était la fille unique de Joseph-Magloire Riou (18611931), véritable seigneur après l'abolition et jusqu'à sa mort en 1931. Son grand-père, Éloi Riou (1798-1864), seigneur au moment de l'abolition et premier maire de Trois-Pistoles, était né à la fin du XVIII ${ }^{e}$ siècle. Les mariages tardifs du père et du grand-père d'Anita Riou ont eu pour résultat une situation exceptionnelle où à peine trois générations permettent de traverser une période de quatre siècles. Cet ancrage à l'histoire des lieux, cette filiation aux derniers seigneurs légitimait, aux yeux de la population locale, une désignation honorifique d'une autre époque. À cette reconnaissance symbolique s'ajoutait certainement le statut de grands propriétaires terriens de la famille, vestige de l'ancien domaine seigneurial et des terres non concédées au moment de l'abolition.

Dans son entretien, Anita Rioux accorde cependant une valeur assez négligeable au statut de sa famille et répète qu'elle n'aimait pas se faire appeler "seigneuresse». "Ça riait!», avance-t-elle en guise d'explications ${ }^{70}$. Son fils Gaston, cadet de la famille, également rencontré en entrevue, voit les choses différemment. Confirmant l'humilité de sa mère, il lance: «Pour moi, c'est de la notoriété ${ }^{1}$. » Dans un coin de pays où les Rioux sont légion, on le surnomme parfois encore le «seigneur» et lui-même se désigne fièrement comme «le fils de la seigneuresse»: «Certaines personnes ne connaissent même pas mon nom ${ }^{72}$.» Fier des réalisations de sa mère, qui fut longtemps présidente de la Commission scolaire régionale, il est cependant conscient que cette histoire est en voie d'être oubliée. Propriétaire du dernier vestige du domaine seigneurial, la "rue de la seigneurie», où il possède encore quelques terrains, il n’hésite pas à dire que les plus jeunes, même ses propres enfants, ignorent les questions de seigneurie ${ }^{73}$.

Ailleurs, les témoins, plus jeunes, se souviennent parfois de ces titres portés par une mère, une grand-mère, pratiques corroborées par d'autres témoins indirects ou sources écrites. Si ces titres et même le souvenir de ceux-ci s'estompent, d'autres témoignages de l'altérité seigneuriale sont revenus fréquemment lors des entretiens, attestant d'une distance physique et sociale face à la population. Yves La Roque de Roquebrune, qui

68. Benoît Grenier, "Jean Rioux : émigrant breton, seigneur canadien», Annales de Bretagne et des Pays de l'Ouest, 111, 2 (été 2004), p. 73-88.

69. $\mathrm{M}^{\mathrm{me}}$ Rioux écrivait son nom sans le $\mathrm{x}$ final jusqu'à son mariage avec son «cousin», un Rioux avec x. "C'était bien important!", nous a-t-elle confié lors de son entretien.

70. Entretien 2015-03 avec Anita Rioux, Trois-Pistoles, 15 mai 2015.

71. Entretien 2015-04 avec Gaston Rioux, Trois-Pistoles, 15 mai 2015.

72. Entretien 2015-04 avec Gaston Rioux, Trois-Pistoles, 15 mai 2015.

73. Entretien 2015-04 avec Gaston Rioux, Trois-Pistoles, 15 mai 2015. 
a passé les étés de son enfance au manoir avec ses grands-parents à SainteCatherine-de-Fossambault, dépeint de manière particulièrement éloquente ce rapport d'Ancien Régime:

On était [mon frère et moi] les petits seigneurs au manoir. [...]Je me souviens quand on allait au village avec grand-papa pour aller chercher la poste ou aller à la boulangerie, on était des petits Monsieurs ${ }^{74}$ ! Donc, il y avait cette distance qui était... concrète et qui était respectée de part et d'autre. Quand Elzéar venait porter la crème en charrette ou le beurre dans les moules, il aurait été extrêmement inconfortable si grand-maman l'avait invité à entrer dans la cuisine. Il fallait vraiment respecter son rôle ${ }^{75}$.

C’est largement par les honneurs réservés à la famille seigneuriale que se manifeste le rapport d'altérité dans la localité ${ }^{76}$. Or, les droits honorifiques ont été officiellement abolis par la loi de 1854. C’est d'ailleurs le seul droit seigneurial qui ne reçut aucune indemnisation ${ }^{77}$. Néanmoins, ces pratiques se sont poursuivies dans bien des cas, notamment avec le consentement des fabriques de paroisses. Déjà, Philippe Aubert de Gaspé, nostalgique de la fin du régime à laquelle il assistait, le mentionnait dans ses écrits: «Lors de l'abolition de la tenure seigneuriale, il y a neuf ans, les marguilliers de l'œuvre et fabrique de la paroisse de Saint-Jean-Port-Joli décidèrent que, nonobstant l'acte du parlement à ce contraire, je jouirais du banc seigneurial ma vie durant ${ }^{78}$. " La famille Aubert de Gaspé ne se maintiendra pas dans la région de Port-Joli et le manoir sera incendié au début $\mathrm{du} \mathrm{XX}^{\mathrm{e}}$ siècle. Toutefois, aux quatre coins du Québec, les témoins ont corroboré les dires du seigneur et auteur et se souviennent des honneurs conférés à leur famille, en particulier à l'église. Yves La Roque de Roquebrune se rappelle, enfant, l'odeur de l'encens dont on aspergeait tout particulièrement ses grandsparents dans l'église de Sainte-Catherine et qui lui causait la nausée. «Je finissais toujours sur le perron en train de vomir ${ }^{79}$ ", raconte-t-il en riant. À Trois-Pistoles, Anita Rioux évoque les trois bancs dont la famille a continué de bénéficier pendant de longues années, tradition disparue à la fin du $\mathrm{XX}^{\mathrm{e}}$ siècle. "Ça dépendait du curé», se souvient-elle ${ }^{80}$. À Saint-Georges-de-

74. Entretien 2015-07 avec Yves La Roque de Roquebrune, Montréal, 16 juin 2015.

75. Entretien 2015-07 avec Yves La Roque de Roquebrune, Montréal, 16 juin 2015.

76. La similitude avec les observations consignées par Éric Mension-Rigau à propos des châtelains de la France actuelle est frappante: É. Mension-Rigau, La vie des châteaux..., p. 48 et ss.

77. Acte seigneurial, art. 14.

78. Philippe Aubert de Gaspé, Les Anciens Canadiens (Québec, Desbarats et Derbishire, 1863), p. 285, deuxième note.

79. Entretien 2015-07 avec Yves La Roque de Roquebrune, Montréal, 16 juin 2015.

80. Entretien 2015-03 avec Anita Rioux, Trois-Pistoles, 15 mai 2015. 
Beauce, la famille Pozer, pourtant anglicane, possédait son banc dans l'église paroissiale catholique. Charlotte Pozer, «la petite Charlotte», 85 ans, venue de la région de Toronto jusqu’à Saint-Georges pour répondre à nos questions, insiste sur le fait que même s'ils étaient protestants, ils avaient leur banc à l'église catholique ${ }^{81}$. S'ils n'allaient pas à la messe très souvent parce qu'ils avaient leur chapelle ainsi que leur cimetière, ils ont maintenu ce droit «seigneurial» jusque dans sa jeunesse. Aussi, se souvient-elle d'une messe de Noël autour des années 1930-1940 où, jeune fille, elle avait eu une altercation avec un paroissien qui lui contestait ce privilège, ne sachant pas qui elle était ${ }^{82} \ldots$ Les quelques familles protestantes n’ont pas évoqué - c'est même plutôt le contraire - d'animosité associée à cette altérité religieuse. Ici, la mémoire semble idéaliser les relations sociales paroissiales et oublier les conflits, pourtant nombreux, relatifs à cette dimension tout au long du XIX $^{\mathrm{e}}$ siècle ${ }^{83}$.

Non seulement a-t-on mémoire de ces privilèges, mais l'enquête a permis de constater que certaines communautés continuent d'attribuer ces honneurs aux descendants de la famille seigneuriale, notamment lorsqu'ils décèdent. À Vaudreuil, où subsiste un patrimoine religieux de grande valeur, l'église Saint-Michel a encore sa chapelle seigneuriale sous laquelle reposent les membres de la famille. Des stèles funéraires ornent les murs et le banc seigneurial est flanqué des armoiries de la famille. Ce banc est encore occupé par une descendante de la famille et lorsqu'elle ne va pas à la messe, personne ne s'y assoit, nous a rapporté l'abbé Normand Bergeron, curé de Vaudreuil ${ }^{84}$. La famille seigneuriale y fait aussi revivre les traditions annuellement lors de la messe des Seigneuriales de VaudreuilDorion, "A historical re-enactment ${ }^{85}$ " et lors du souper des seigneurs ${ }^{86}$. À Lotbinière, on retrouve également un banc seigneurial, plus simple, mais aussi orné des armoiries de la famille (qui s'est scindée en deux branches

81. Entretien 2017-04 avec Charlotte Pozer-Gamble, Saint-Georges-de-Beauce, $1^{\text {er }}$ août 2017.

82. Entretien 2017-04 avec Charlotte Pozer-Gamble, Saint-Georges-de-Beauce, $1^{\text {er }}$ août 2017.

83. Les rapports des seigneurs protestants aux paroisses catholiques, en particulier l'usage du banc seigneurial, mériteraient de faire l'objet d'une étude plus attentive dans une perspective historienne. On retrouve bien des allusions éparses à cet enjeu, souvent dans des travaux d'histoire locale, mais aucune étude ne s'y est spécifiquement consacrée. Voir, par exemple: Paul-Henri Hudon, «Le temps des paroissiens", Les Cahiers de la seigneurie de Chambly, 37 (septembre 2013), p. 36 ou encore J. I. Little, Patrician Liberal..., p. 95.

84. Entretien 2016-02 avec l'abbé Normand Bergeron, Vaudreuil,15 avril 2016.

85. C'est ainsi que Peter de Lotbinière-Harwood définit l'événement qui se tient annuellement au début du mois de juin. Entretien 2016-07 avec Peter de Lotbinière-Harwood, Vaudreuil, 4 juin 2016. À propos des Seigneuriales, Les seigneuriales de Vaudreuil-Dorion, : <http://seigneuriales.com/>.

86. Voir le court-métrage consacré au cas de Vaudreuil : Persistances et mémoires seigneuriales au Québec: l'exemple de la seigneurie de Vaudreuil (2017), S. Lanthier réalisatrice: < https:/ / youtu.be/Pjyf2Sp6P2s>. 
Figure 3

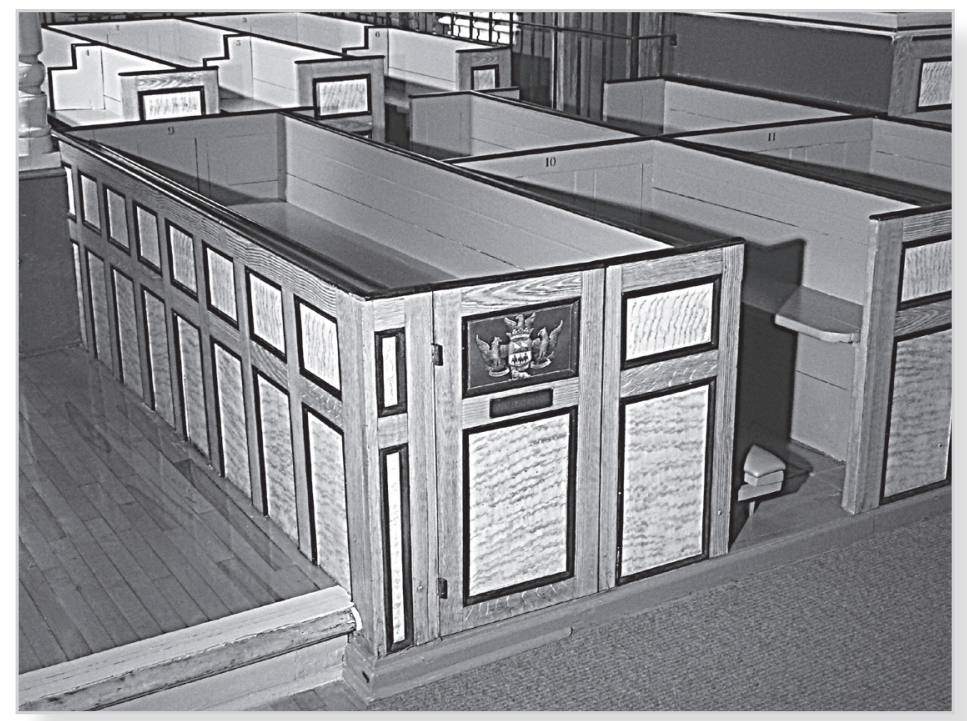

Banc seigneurial et armoiries dans l'église Saint-Michel-de-Vaudreuil

Crédit: Benoît Grenier

principales au XIX ${ }^{\mathrm{e}}$ siècle), où venait s'asseoir, au moins quelques fois l'an, "Monsieur Edmond», qui séjournait l'été dans l'ancienne seigneurie familiale ${ }^{87}$.

Comme Philippe Aubert de Gaspé, de nombreux seigneurs reposent sous leur banc seigneurial dans les anciennes églises du Québec. Cette pratique est toujours en vigueur dans un certain nombre de localités, dont deux de celles que nous avons visitées dans le cadre de la recherche: Terrebonne et Vaudreuil ${ }^{88}$. En fait, il semble bien que ce soit la généralisation de l'incinération et de l'inhumation des cendres qui ait contribué à faire revivre cette pratique, notamment pour des raisons d'espace, comme en témoigne le "sarcophage» de béton de la «Famille Harwood» sous la chapelle Saint-Louis de l'église de Vaudreuil. Les témoignages de différents membres de la famille Lotbinière-Harwood, de même que celui du curé, nous ont appris que la pratique est bien actuelle et que la dernière

87. Rapporté par des membres de la Société d'histoire des seigneuries de Lotbinière. Les Joly de Lotbinière se sont défaits du "domaine Joly de Lotbinière " en 1972 (notice nécrologique de 2014), mais le banc seigneurial subsiste en l'église Saint-Louis.

88. É. Mension-Rigau dresse un pareil constat pour la France dans La vie des châteaux..., p. 48-49. 
inhumation a eu lieu en $2013^{89}$. Robert de Lotbinière-Harwood a déjà exprimé le souhait d'y rejoindre ses ancêtres le moment venu, tandis que son cousin Peter, résidant de Vancouver, y songe ${ }^{90}$. À Terrebonne, le soussol de l'église est une véritable nécropole de la famille Masson. Comme à Vaudreuil, des inhumations y ont eu lieu au cours des années $2000^{91}$. Qui plus est, en cours de recherche, nous apprenions le décès du propriétaire du manoir de Saint-Ours-sur-le-Richelieu, héritier des seigneurs de l'endroit et on pouvait lire dans le journal local que «[ses] cendres $[\ldots]$ seront inhumées dans la crypte du sous-sol de l'église paroissiale, quelques semaines plus tard. $\mathrm{M}^{\mathrm{e}}$ Poupart rejoindra ainsi d'autres descendants de son illustre ancêtre qui s'y trouvent déjàà . » À Sainte-Catherine-de-laJacques-Cartier, dont l'église est plus récente, reconstruite à la suite d'un incendie au $\mathrm{XX}^{\mathrm{e}}$ siècle, c'est dans le vieux cimetière dit des «JuchereauDuchesnay» que les membres de la famille sont inhumés encore de nos jours. Gardons-nous d'y voir une proximité plus grande avec les autres paroissiens de l'endroit, puisque les différents cousins issus des sœurs Clara et Louise Juchereau-Duchesnay - dernières du nom à cet endroit - sont inhumés au pied de la grande croix au centre du cimetière, rappel éminent de la distinction de cette famille ${ }^{93}$. Même Anne Hébert, au terme d'une vie passée essentiellement à Paris, demanda à y reposer, après des obsèques en toute intimitée ${ }^{94}$.

\section{Une conscience de soi dans la durée}

En plus de l'appartenance au terroir seigneurial, les familles rencontrées révèlent une conscience d'elles-mêmes dans la durée, plusieurs se revendiquant d'ailleurs de l'ancêtre de la lignée. À l'heure où la pratique de la généalogie est de plus en plus répandue, il n’est pas toujours aisé de distinguer ce qui relève de recherches et ce qui vient de la tradition familiale $^{95}$. À l'instar d'Edmond Joly, dont la nécrologie évoque Louis XIV et

89. Entretien 2016-02 avec l'abbé Normand Bergeron, Vaudreuil, 15 avril 2016.

90. Entretiens 2016-07 avec Robert Chartier de Lotbinière-Harwood et 2016-07 avec Peter de Lotbinière-Harwood, Vaudreuil, 4 juin 2016.

91. Entretien 2016-05 avec Claude Martel, président de la Société d'histoire de la région de Terrebonne, 29 avril 2016.

92. Louise Grégoire-Racicot, "Saint-Ours met ses drapeaux en berne pour Me Armand Poupart», Les Deux Rives. La Voix, 25 mars 2016.

93. Entretien 2016-01 avec Michel Fragasso, Longueuil, $1^{\mathrm{er}}$ avril 2016.

94. Entretien avec Nathalie Watteyne dans le cadre du documentaire L'attachement seigneurial de l'écrivaine Anne Hébert (2017) réalisé par S. Lanthier: < https://youtu.be/bsTdwlzmB0A>. Voir aussi: Nathalie Watteyne et Bernard Chassé, Album Anne Hébert (Montréal, Fides, 2016).

95. Le rôle de la généalogie pour les familles de la noblesse n'a cependant rien de nouveau. Plusieurs études fort intéressantes y ont été consacrées: Germain Butaud et Valérie Pietri, Les enjeux de la généalogie 
Jean Talon ainsi que le premier ancêtre de la lignée canadienne, les familles rencontrées, lorsque questionnées sur leurs origines familiales, ont régulièrement mis de l'avant leur "ancienneté» sur place, en rappelant le souvenir de leur premier ancêtre devenu seigneur. Sans nécessairement pouvoir détailler toute la chaîne généalogique jusqu'à eux, ils avaient clairement conscience de l'importance de cette antériorité : «La mémoire du passé est essentielle : conditionnant les pratiques sociales et marquant sociologiquement les distances, elle demeure le repère identitaire le plus efficace ${ }^{96} »$, rappelle à juste titre Éric Mension-Rigau. Cette conscience se double d'une évidente fierté envers leurs ancêtres "fondateurs", une fierté qui se comprend du fait que les témoins rencontrés sont, en quelque sorte, eux-mêmes les traces vivantes de cette durée. Il y a fort à parier que d'autres membres de ces familles, qui n'ont pas entretenu de tels liens avec la "seigneurie», ne seraient pas en mesure de fournir les mêmes renseignements ni d'en tirer une telle fierté. Le souvenir de Jean Riou et de Catherine Leblond est évoqué par Anita Rioux à Trois-Pistoles: "on est ici depuis $16 \ldots 1600$ quelque chose ${ }^{97}$ », rappelle-t-elle. Pour les Pozer, descendants d'une famille allemande, la mémoire de George Pfozer, épicier devenu seigneur en Beauce, est aussi source de fierté pour avoir été le fondateur de ce qui deviendra, en son honneur, Saint-Georges ${ }^{98}$. D’ailleurs, comme ils se plaisent à le rappeler, les désignations de paroisses et les toponymes évoquent souvent le souvenir de ces ancêtres fondateurs. Pour Liane de Lotbinière-Harwood, aujourd'hui résidant à Toronto, c'est un privilège d'appartenir à une famille dont on peut remonter les racines jusqu'au Moyen Âge ${ }^{99}$. Semblable sentiment de fierté pour Yves de Roquebrune qui nous présente une copie des lettres de noblesse accordées à l'ancêtre Nicolas Juchereau de Saint-Denys ${ }^{100}$. Cette mémoire des aïeux passe aussi par un rapport particulier à l'histoire du Canada et du Québec dont ces familles sont souvent indissociables ${ }^{101}$. Les souvenirs d'enfance

(XII'-XVIII siècles). Pouvoir et identité (Paris, Autrement, 2006); Olivier Rouchon, L'opération généalogique. Cultures et pratiques européennes, $X V^{e}$-XVIII siècles (Rennes, Presses universitaires de Rennes, 2014). Voir aussi l'exemple de la famille Salaberry bien étayé dans la thèse de V. Chaleur-Launay, «Les Salaberry entre deux empires...».

96. É. Mension-Rigau, La vie des châteaux..., p. 337.

97. Entretien 2015-03 avec Anita Rioux, Trois-Pistoles, 15 mai 2015.

98. Entretien 2017-06 avec Gordon Pozer, Saint-Georges-de-Beauce, $1^{\mathrm{er}}$ août 2017.

99. Entretien 2016-09 avec Liane de Lotbinière-Harwood, Vaudreuil, 4 juin 2016.

100. Entretien 2015-07 avec Yves La Roque de Roquebrune, Montréal, 16 juin 2015.

101. Le père Benoît Lacroix se souvenait de ses visites au manoir de Fossambault où la "seigneuresse» Hermine Prévost exaltait la mémoire de ses ancêtres : «Les parents de Saint-Denys Garneau », Les Cahiers Anne-Hébert, 7 (2007), p. 11-17. 
révèlent aussi le mélange d'admiration et de frayeur que pouvaient produire les tableaux des ancêtres conservés dans la maison familiale: «Sophie [la seigneuresse Sophie Raymond-Masson], elle nous faisait peur!», se souvient Amélie Masson-Labonté ${ }^{102}$, sentiment qu'elle partage avec Liane de Lotbinière à propos des portraits de la famille de Lotbinière ${ }^{103}$.

Cette mémoire des ancêtres n’est pas que "généalogique», comme en témoigne la transmission des patronymes et des prénoms distinctifs au fil des siècles. Ainsi, le prénom De Saint-Denys, rappelant le nom de l'ancêtre Juchereau anobli, revient à de nombreuses reprises dans les différentes branches de la lignée. Michel Biron, biographe de De Saint-Denys Garneau, souligne que ce prénom peu commun: "trahit cet orgueil familial, fondé sur le culte de la filiation. Il vient de l'ancêtre Nicolas Juchereau, dont le patronyme "de Saint-Denys" est évoqué dès 1670 dans les Relations des Jésuites. [...] le fait d'attribuer un tel prénom à un enfant en 1912 en dit long sur la personnalité d'Hermine et sur son ascendant au sein de la famille ${ }^{104}$ ». Hermine Prévost elle-même porte un prénom transmis par les familles Salaberry et Juchereau-Duchesnay dont elle est descendante ${ }^{105}$. Pauline Garneau, sœur du poète, nommera à son tour l'un de ses fils De SaintDenys. Chez les Pozer, on est Georges de père en fils, tandis qu'Henri est incontournable pour les Masson.

Chez les Lotbinière-Harwood, les fils se prénomment Charles, Henri ou Alain, comme le rappelle Liane: «Je trouvais ça toujours un petit peu particulier quand j'étais petite, j'ai mon grand-père Charles, mon papa Charles, mon cousin Charles... qu'est-ce qui se passe avec ça ${ }^{106}$ ?» et la perpétuation du patronyme, notamment pour les familles d'ascendance noble, peut-être plus conscientes de leur distinction, n'est pas moins importante. Quoi que certains prénoms féminins soient récurrents, il ne semble pas y avoir un souci aussi marqué à perpétuer les prénoms des femmes. Toutefois, le nom «de Lotbinière» s'est maintenu grâce aux femmes et à leur époux (Joly et Harwood), qui ont bien compris, au $\mathrm{XIX}^{\mathrm{e}}$ siècle, la valeur ajoutée de ce patronyme à particule en devenant respectivement des Joly de Lotbinière et des Lotbinière-Harwood ${ }^{107}$. La plus jeune de nos témoins, Poème de Lotbinière-Carvalho, 8 ans, née à

102. Entretien 2016-06 avec Amélie Masson-Labonté, Terrebonne, 29 avril 2016.

103. Entretien 2016-09 avec Liane de Lotbinière-Harwood, Vaudreuil, 4 juin 2016.

104. M. Biron, De Saint-Denys Garneau..., p. 19.

105. V. Chaleur-Launay, "Les Salaberry entre deux empires... », p. 459-460.

106. Entretien 2016-09 avec Liane de Lotbinière-Harwood, Vaudreuil, 4 juin 2016.

107. J. I. Little, Patrician liberal..., p. 46. 
Toronto, porte le patronyme de sa mère, attachée à sa lignée malgré son éloignement géographique ${ }^{108}$.

On l'aura compris, les individus rencontrés dans le cadre de cette enquête témoignent d'une identité façonnée, à des degrés divers, par leurs origines seigneuriales: un attachement à la terre ancestrale, le maintien jusqu'à une période somme toute récente d'une forme d'altérité se manifestant par des honneurs et distinctions à l'échelle locale et un sens de l'histoire et des origines, bien plus que par une aisance matérielle. Cette identité vient aussi avec certaines valeurs distinctives qui ont été transmises jusqu'à nos jours, bien qu’elles se soient atténuées au cours du dernier demi-siècle.

\section{DES VALEURS DISTINCTIVES?}

De cet héritage seigneurial découlent des valeurs «nobiliaires» qui sont clairement énoncées par plusieurs des témoins rencontrés. Sans chercher à catégoriser de manière trop radicale, nous avons cru déceler un certain nombre de traits communs qui lient ces familles. Nous noterons cependant, à cet égard, des nuances entre les familles d'origine noble et celles d'ascendance roturière. La première incarne peut-être, justement, ce que les premières désignent comme le «sens de la noblesse».

\section{Noblesse oblige!}

C'est ainsi qu'Yves La Roque de Roquebrune a résumé les valeurs qui lui avaient été transmises enfant ${ }^{109}$. L'honneur constitue, on le sait, une composante centrale de l'identité nobiliaire à l'époque moderne, en France comme en Nouvelle-France ${ }^{110}$. Quoique tous les seigneurs de la vallée du Saint-Laurent n'aient pas appartenu à la noblesse et que la valeur juridique de celle-ci soit fortement discutable après le changement de régime et certainement devenue désuète au tournant du $\mathrm{XX}^{\mathrm{e}}$ siècle, il faudra mieux étudier l'ensemble du groupe nobiliaire pour saisir ce que signifie l'appartenance à ce groupe social ${ }^{11}$. Plusieurs témoins ont revendiqué cet héritage familial. Pour Yves de Roquebrune, comme pour son lointain cousin

108. Entretien 2016-10 avec Poème de Lotbinière-Carvalho, Vaudreuil, 4 juin 2016.

109. Entretien 2015-07 avec Yves La Roque de Roquebrune, Montréal, 16 juin 2015.

110. Frédérique Leferme-Falguières, «La noblesse de cour aux XVII ${ }^{\mathrm{e}}$ et XVIII ${ }^{\mathrm{e}}$ siècles. De la définition à l'autoreprésentation d'une élite», Hypothèses, 1-4 (2001), p. 87-98. Les travaux d'Ollivier Hubert sur l'honneur et la masculinité sont également à connaitre.

111. Le travail du généalogiste Yves Drolet, en particulier son Dictionnaire généalogique de la noblesse de la Nouvelle-France, permet d'envisager une telle étude. Karine Pépin réalise actuellement un doctorat sur cette question, en cotutelle à l'Université de Sherbrooke et à Sorbonne-Université. Voir aussi la communication inédite de Y. Drolet, «La brunante des gentilshommes: la noblesse canadienne à l'ère de la démocratie bourgeoise » qu'il m’a aimablement communiquée. 
Michel Fragasso, cette appartenance venait avec un mode de vie et des habitus dignes de leur nom et de leur rang: niveau de langue approprié, manières à table, apparence physique, code vestimentaire, etc. En parlant de sa grand-mère, issue des Juchereau-Duchesnay, Michel Fragasso nous dit: "elle voulait que je sois peigné comme le prince Charles ${ }^{112}$ ». Henri Masson fils, issu d'une famille seigneuriale bourgeoise très influente au tournant du XX $\mathrm{XX}^{\mathrm{e}}$ siècle $^{113}$, évoque aussi ce "décorum» qui accompagnait leur éducation ${ }^{114}$. Yves de Roquebrune, encore, rappelle qu’il leur était interdit de jouer avec les autres enfants du village à Sainte-Catherine ${ }^{115}$.

Autre vestige du sens de la noblesse : le rapport ambigu à l'argent évoqué par plusieurs témoins en parlant de leur famille. Après tout, le noble ne doit pas travailler, au risque de déroger; c'est l'essence même de la noblesse d'Ancien Régime. Plusieurs héritiers de familles seigneuriales ont fait des études (droit et médecine surtout), sans jamais exercer leur profession, vivant, en quelque sorte, "noblement» et en se consacrant parfois aux affaires publiques. Dans la préface qu'elle signe du journal de Fadette (Henriette Dessaules - 1860-1946) - sa grand-mère - Louise Dechêne écrit: «Les frères Dessaules ont étudié, l’aîné la médecine, l’autre le droit [...]. Ces études sont considérées comme simples instruments de culture car ni l'un ni l'autre n'a même tenté d'exercer sa profession ${ }^{116}$.» Pour ceux qui "travaillent», on remarque des traditions professionnelles familiales dignes de leur rang, en quelque sorte une culture familiale de service de l'État, ou plus exactement de service du roi (ou de la reine!): service militaire, fonction publique, diplomatie, magistrature... dans la droite lignée de ce qu'avançait L.-A. Taschereau en $1922^{117}$. Faut-il s'étonner de retrouver Charles de Lotbinière-Harwood chef du protocole lors des Jeux Olympiques de Montréal de 1976, au plus près de la reine Élizabeth II, venue inaugurer

112. Entretien 2016-01 avec Michel Fragasso, Longueuil, $1^{\mathrm{er}}$ avril 2016.

113. Sur la famille Masson: Andrée Désilets, Louis-Rodrigue Masson. Un seigneur sans titres (Montréal, Boréal Express, 1985), ainsi que Thierry Nootens, "Je crains fort que mon pauvre Henri ne fasse pas grand chose..." ", Revue d'histoire de l'Amérique française, 61, 1 (été 2007), p. 223-257. Henri Masson, père du témoin rencontré, a lui-même, fait œuvre d'historien amateur en publiant, à compte d'auteur, une histoire du seigneur Joseph Masson: Joseph Masson, dernier seigneur de Terrebonne 1791-1847 (s.1, s.e., 1972).

114. Entretien 2017-01 avec Henri Masson, Ottawa, 21 avril 2017.

115. Entretien 2015-07 avec Yves La Roque de Roquebrune, Montréal, 16 juin 2015.

116. Louise Saint-Jacques Dechêne, «Introduction», Fadette. Journal d'Henriette Dessaules 1874-1880 (Montréal, Hurtubise HMH, 1971), p. 15. Dans cette introduction, l'auteure ne mentionne pas son appartenance à cette famille. Rappelons que les Dessaules, pas plus que leurs alliés Papineau, n'appartiennent à la noblesse. Bien que bourgeoise, c'est une famille d'origine roturière, témoignant de la fluidité entre ces groupes au XIX ${ }^{\mathrm{e}}$ siècle.

117. Louis-Alexandre Taschereau, "La noblesse canadienne...». 
les Jeux ${ }^{118}$ ? Ces habitus seigneuriaux, ce «naturel» d'autorité, qui leur ont été transmis sont, à des degrés divers, bénéfiques dans leur propre vie. Plusieurs des descendants rencontrés, et plus encore leurs parents, sont héritiers d'un capital symbolique qu'ils ont su mettre à profit. L'un des témoins rencontrés a cité pas moins de deux premiers ministres du Québec parmi ses «intimes».

Ce comportement de rentiers du sol n'est pas sans poser problème parvenu au $\mathrm{XX}^{\mathrm{e}}$ siècle, alors que la seigneurie ne rapporte plus guère de revenus très substantiels, sauf peut-être pour des familles qui ont préservé d'importantes propriétés foncières qu'elles peuvent lotir ou exploiter à d'autres fins. Mais cela n'est pas le cas de toutes les familles et plusieurs ne semblent pas nécessairement dotées d'un sens des affaires très aiguisé ${ }^{119}$. Ces problèmes d'argent, dont on ne parle évidemment pas en société, sont d'autant plus embêtants qu'il faut maintenir un mode de vie digne du rang revendiqué, surtout lorsqu'on possède un manoir et, très souvent, une résidence à Québec ou à Montréal. Si certaines familles semblent à l'abri de ces soucis (c'est le cas des héritiers Masson par exemple ${ }^{120}$ ), d'autres sont véritablement embarrassées. Yves La Roque de Roquebrune se souvient que ses grandsparents étaient relativement "pauvres», mais devaient entretenir un vaste domaine et vivre comme des aristocrates: «Il va falloir manger de la mélasse», se souvient-il avoir entendu dire son grand-père pour signifier qu'ils devraient se serrer la ceinture afin d'assumer leurs obligations «seigneuriales». Sa grand-mère finira par devoir louer des chambres dans leur maison de Westmount ${ }^{121}$, confie-t-il. De telles situations, sans être misérables et qu'il faut certainement relativiser, conduiront cependant bon nombre de familles à se départir du manoir seigneurial.

Autre vestige des pratiques nobiliaires, les anciennes familles seigneuriales, surtout celles appartenant à la vieille aristocratie terrienne, ont longtemps maintenu une forme d'endogamie familiale ${ }^{122}$. Les liens sont

118. Entretien 2016-07 avec Robert Chartier de Lotbinière-Harwood, Terrebonne, 4 juin 2016,

119. C'est le cas des Dessaules, à Saint-Hyacinthe, qui se départissent du domaine au profit de T.-D. Bouchard en 1911, lequel, plus avisé, lotira ces terres où l'on retrouve aujourd'hui le centre-ville de Saint-Hyacinthe: Michel Morissette, «L'abolition des droits seigneuriaux : une affaire d'argent», Cap-auxDiamants, 112 (hiver 2013), p. 27-30.

120. Entretien 2016-06 avec Amélie Masson-Labonté, Terrebonne, 29 avril 2016.

121. Entretien 2015-07 avec Yves La Roque de Roquebrune, Montréal, 16 juin 2015. Il évoque une «fête paroissiale quelconque», coûteuse pour la famille, lors de laquelle on recevait les habitants au manoir de Sainte-Catherine.

122. Lorraine Gadoury, La noblesse de Nouvelle-France. Familles et alliances (Montréal, Hurtubise HMH, 1992); Karine Pépin, «Mariage et altérité: Les mariages mixtes chez la noblesse canadienne après la Conquête (1760-1800)", mémoire de maîtrise (histoire), Université de Sherbrooke, 2016. 
étroitement imbriqués entre plusieurs d'entre elles, auxquelles s'intégreront de nouveaux venus issus de la bourgeoisie anglaise ou écossaise - tels les Campbell de Rouville - au moins jusqu'au tournant du XX $\mathrm{X}^{\mathrm{e}}$ siècle. Le généalogiste Yves Drolet, auteur d'un Dictionnaire des familles nobles de la Nouvelle-France, parle du «dernier carré de la noblesse canadienne, formé au XIX ${ }^{\mathrm{e}}$ siècle par des mariages entre les Salaberry, Juchereau-Duchesnay, Saint-Ours, Hertel de Rouville et Taschereau ${ }^{123}$ ». Il est vrai que la généalogie de certains de nos témoins constitue à n'en pas douter un condensé de l'histoire du Québec. La mémoire de ces alliances s'effrite cependant chez les descendants qui ignoraient souvent ces ramifications complexes ${ }^{124}$. De toute évidence, à la différence du cas français où l'endogamie de classe persisterait, de telles pratiques n'ont plus cours au Québec parmi les descendants de seigneurs ${ }^{125}$.

\section{Une culture familiale du bilinguisme}

Il est un trait très présent chez presque toutes les familles rencontrées et qui peut certainement être interprété comme une originalité du groupe seigneurial canadien, c'est le bilinguisme. Pour les familles issues de l'ancienne aristocratie (Juchereau-Duchesnay, Joly de Lotbinière, LotbinièreHarwood, Aubert de Gaspé...), l'importance du bilinguisme semble étroitement liée à leur héritage familial, remontant souvent au lendemain même de la Conquête, alors qu'elles vont accepter de servir le nouveau maître du pays ${ }^{126}$. Cela passe aussi bien souvent dans ces familles par une tradition d'alliances mixtes qui se répercute jusqu'à nos jours et dont la famille Lotbinière-Harwood (issue d'une union mixte contractée au milieu du XIX ${ }^{e}$ siècle) semble particulièrement représentative ${ }^{127}$. Ce bilinguisme, voire ce biculturalisme, les rend aussi à l'aise à Vancouver qu'à Québec et les situe, à la suite de leurs ancêtres, dans une perspective canadienne, souvent fervents défenseurs de l'unité canadienne, poursuivant en quelque sorte dans la voie pavée par leurs aïeux qui ont choisi l’Angleterre plutôt que la France. Ainsi, de rappeler le traducteur (F.R.)

123. Entretien 2017-08 avec Yves Drolet, Montréal, 8 décembre 2017.

124. À titre d'exemple, sachant qu'une fille Masson (Marie-Adélaïde) avait épousé l'héritier Lotbinière-Harwood (Charles-Auguste) à la fin du XIX ${ }^{\mathrm{e}}$ siècle, nous avons simplement glissé l'anecdote en cours de conversation, mais les témoins des deux familles l'ignoraient, malgré une stèle encore bien visible dans l'église Saint-Michel de Vaudreuil où reposent les deux époux. Une étude plus approfondie

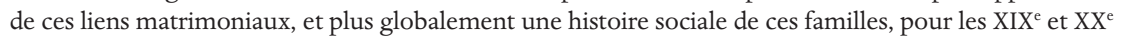
siècles est en cours.

125. É. Mension-Rigau, La vie des châteaux..., p. 327.

126. Sur la noblesse après la Conquête: Sophie Imbeault, Les Tarieu de Lanaudière. Une famille noble après la Conquête 1760-1791 (Québec, Septentrion, 2004).

127. K. Pépin, «Mariage et altérité...», p. 171. 
du discours de L.-A. Taschereau cité en amorce de ce texte: «ces deux discours ont été prononcés en anglais, langue que l'hon. M. Taschereau parle avec autant d'élégance que de facilité. C'est une leçon de bilinguisme qui s'est ajoutée délicatement aux arguments de l'orateur. Le texte que nous publions maintenant n'est qu'une traduction aussi fidèle que possible ${ }^{128}$." D'autres familles, de tradition seigneuriale «bourgeoise» plus récente, ayant accédé à la propriété de fiefs au XIX ${ }^{\mathrm{e}}$ siècle (Masson, Pozer, Campbell) ont aussi évoqué cette aisance à naviguer aussi bien dans le monde francophone que dans le monde anglophone. Il s'agit d'une piste qui mériterait d'être explorée davantage ${ }^{129}$.

\section{Le sens de l'engagement: la contrepartie des privilèges seigneuriaux?}

Le noble, par essence, est un privilégié et, nobles ou roturiers, les seigneurs sont aussi les privilégiés d'un système fondé sur les inégalités propres à l'Ancien Régime. Sans vouloir revenir ici sur le débat historiographique relatif à la nature utilitaire ou parasitaire du régime seigneurial canadien $^{130}$ ni prendre fait et cause pour l'interprétation trudélienne des droits et devoirs réciproques ${ }^{131}$, force est de constater que l'histoire que relatent les descendants de seigneurs, on ne s'en étonnera sans doute pas, est celle des seigneurs défricheurs, ou du moins des seigneurs-fondateurs, loin des courtisans de Versailles ${ }^{132}$. «Entre nous, c'est des seigneurs, mais pas des aristocrates [rire], ce sont nos seigneurs à nous!», affirme d'entrée de jeu Marie-José Raymond ${ }^{133}$. On notera que la descendante des Dessaules de Saint-Hyacinthe fait une lecture bien différente de celle de sa cousine historienne, Louise Dechêne ${ }^{134}$.

128. Préface de L'habitant de Québec \& Noblesse Canadienne-Française, conférences prononcées à Toronto devant l'Empire club et le Women's Canadian Club le 27 avril 1922, par l'honorable L.-A. Taschereau.

129. L'attitude des élites face à ce biculturalisme a fait l'objet d'un récent ouvrage collectif qui invite à explorer cette avenue: Alex Tremblay-Lamarche et Serge Jaumain, dir., Les élites et le biculturalisme. Québec-Canada-Belgique XIX'-XXe siècles (Québec, Septentrion, 2017).

130. Voir entre autres: Serge Jaumain et Matteo Sanfilippo, «Le régime seigneurial en NouvelleFrance: un débat historiographique», The Register, 2, 5 (1980), p. 226-247 ou Benoît Grenier et Michel Morissette, «Introduction» de Nouveaux regards en histoire seigneuriale..., p. 13-25.

131. M. Trudel, Le régime seigneurial...

132. Pour paraphraser Benjamin Sulte dans son Histoire des Canadiens-français, 1608-1880 (Montréal, Wilson \& cie, 1882-1884) (vol. 2), p. 104-105.

133. Entretien 2015-01 avec Marie-José Raymond, Saint-Paul d'Abbotsford, 13 février 2015.

134. Louise Dechêne (née Saint-Jacques) était la fille de Louis Saint-Jacques, fils d'Henriette Dessaules et de Maurice Saint-Jacques, et donc l'arrière-petite-fille de Casimir Dessaules. Elle était par conséquent issue de deux lignées seigneuriales influentes, les Dessaules et les Papineau (Rosalie Papineau, sœur de Louis-Joseph, était seigneuresse de Saint-Hyacinthe par son mariage avec Jean Dessaules). Louise Dechêne fut, en dépit de son bagage familial, la première à proposer une interprétation très antipathique du régime 
À la question, "considérez-vous que votre famille est (ou était) privilégiée?», la totalité des répondants ont répondu par la négative, ou avec force nuances, insistant presque tous sur ce qui leur semblait être la contrepartie de ces "privilèges», c'est-à-dire les «devoirs» du seigneur, soit leur rôle auprès des habitants de la seigneurie. Lorsqu'ils admettent que leur famille était en quelque sorte favorisée, ils précisent du même coup que si leurs parents ou grands-parents (selon leur âge) pouvaient effectivement bénéficier d'une forme de privilèges, tel n'est plus le cas pour la génération née après la Seconde Guerre mondiale. Douglas Cann le dit avec un grand sourire: «No privileges! It's more a sense of contributing [avant d'ajouter en parlant des relations avec les gens du Bic]. Ce sont nos voisins et nos amis $^{135}$. " Amélie Masson-Labonté, après réflexion, avance: «Il s’est passé quelque chose dans les années $1960^{136}$ !», avant de rire elle aussi. Dans la transmission de la mémoire seigneuriale, il semble en effet que les années 1960 aient constitué un passage difficile: idéologies antiautoritaires, circulation d'idéaux de gauche et socialisants, État-providence, transformations des pratiques religieuses issues de Vatican II, etc. Pour la jeune étudiante membre du Rassemblement pour l'indépendance nationale (RIN) qu'était Marie-José Raymond, il n'était assurément pas de bon ton de brandir son héritage seigneurial comme un étendard ${ }^{137}$.

Par contre, toutes ces familles partagent, selon des modalités diverses, ce sens de l'engagement que l'on qualifierait de nos jours de "citoyen", mais qui, à leurs yeux, relève de la continuité de pratiques seigneuriales anciennes. C'est peut-être le constat le plus remarquable de cette enquête : ce sens du "service», du "devoir», parfois perçu comme un héritage propre à la noblesse, revient dans tous les entretiens et s'inscrit comme une vision de continuité depuis leurs ancêtres qui «servaient le roi». Yves La Roque de Roquebrune énonce le sens du devoir qui incombe aux nobles $^{138}$. La descendante des seigneurs de Saint-Hyacinthe évoque l'engagement politique local de la famille Dessaules où «il régnait une atmosphère de responsabilité politique ${ }^{139}$ ». En 1972, Louise Dechêne écrivait d'ailleurs à leur sujet: «les habitudes sont plus lentes à mourir et Louis-

seigneurial, laquelle n'est pas du tout celle évoquée par les témoins, à commencer par sa cousine. Sur la non-prégnance de cette interprétation sur la mémoire collective québécoise, voir: B. Grenier, «Les paradoxes de la mémoire seigneuriale...».

135. Entretien 2015-10 avec Douglas Cann, Le Bic, 7 août 2015.

136. Entretien 2016-06 avec Amélie Masson-Labonté, Terrebonne, 29 avril 2016.

137. Entretien 2015-01 avec Marie-José Raymond, Saint-Paul d'Abbotsford, 13 février 2015.

138. Entretien 2015-07 avec Yves La Roque de Roquebrune, Montréal, 16 juin 2015.

139. Entretien 2015-01 avec Marie-José Raymond, Saint-Paul d’Abbotsford, 13 février 2015. 
Antoine, héritier pour la moitié, et après lui, Casimir, qui partage avec sa sœur Rosalie Laframboise l'autre moitié, continuent à faire figure de seigneurs. Peut-être parce que, non contents de continuer de tirer du sol les honneurs et les rentes, ils investissent dans l'avenir de la région et pèsent sur l'orientation de son opinion ${ }^{140}$. » À une échelle plus modeste, les Rioux à Trois-Pistoles, les Pozer à Saint-Georges ou les Tessier dit Laplante à Beauport exercent tous des fonctions politiques au tournant du $\mathrm{XX}^{\mathrm{e}}$ siècle ${ }^{141}$.

Mais l'engagement de ces familles n'est pas que politique. Plus près de nous, certaines se sont investies dans la sauvegarde du patrimoine local. “C'est peut-être pour ça que je m'occupe de mes petites églises ${ }^{142}$ », sourit Marie-José Raymond qui a par ailleurs dénoncé haut et fort la destruction de la maison Casimir-Dessaules par la municipalité de Saint-Hyacinthe en $2011^{143}$. Henri Masson, 77 ans, rappelle les actions de son père, historien amateur et juriste de formation, dans la préservation de l'île des moulins à Terrebonne - héritage patrimonial de leur ancêtre Joseph Masson et de son épouse Sophie Raymond ${ }^{144}$. En entrevue, les dires de la famille sont corroborés par le président de la Société d'histoire régionale, Claude Martel. Ce dernier se souvient d'ailleurs très bien de l'impression que lui faisait, alors jeune étudiant, cet homme élégant qui avait toute l'attitude d'un "seigneur", dont la manière de parler ${ }^{145}$. Au Bic, Douglas Cann explique son propre engagement citoyen dans la préservation du patrimoine local, notamment sa contribution au comité qui luttait contre un promoteur immobilier de Rimouski ou encore pour éviter que l'autoroute 20 ne passe trop près du village ${ }^{146}$.

Quant à Mme Rioux, c'est avec une grande humilité qu'elle raconte avoir maintenu les pratiques de ses ancêtres envers la paroisse ou la municipalité de Trois-Pistoles en donnant des terrains à plusieurs occasions (écoles, hôpital, nouveau cimetière): "Le maire venait nous voir pour qu'on donne, faque, on donnait ${ }^{147}$. " En contrepartie de ces donations, comme au temps des seigneurs, quelques reconnaissances honorifiques, dont un lot "gratuit» au cimetière pour la famille Rioux. La famille Pozer,

\footnotetext{
140. L. Saint-Jacques Dechêne, «Introduction », Fadette. Journal d'Henriette Dessaules..., p. 15.

141. B. Grenier, «Élites seigneuriales, élites municipales... », p. 57-64.

142. Entretien 2015-01 avec Marie-José Raymond, Saint-Paul d'Abbotsford, 13 février 2015.

143. Isabelle Paré, «La Maison Dessaulles démolie sans avertissement», Le Devoir, 5 juillet 2011.

144. Entretien 2017-01 avec Henri Masson, Ottawa, 21 avril 2017.

145. Entretien 2016-05 avec Claude Martel, Terrebonne, 29 avril 2016.

146. Entretien 2015-10 avec Douglas Cann, Le Bic, 7 août 2015.

147. Entretien 2015-03 avec Anita Rioux, Trois-Pistoles, 15 mai 2015.
} 
dont certains membres continuent de vivre à Saint-Georges-de-Beauce, offre un cas intéressant en tant qu'anglicans, d'origine allemande, dans une région majoritairement catholique et francophone. Cette double altérité ne les a pas empêchés (à l'instar des Campbell du Bic et des Campbell de Rouville) de contribuer à l'édification des institutions paroissiales catholiques. Gordon Pozer se rappelle que les petits protestants étaient les seuls garçons à fréquenter le couvent à Saint-Georges parce que leur ancêtre avait donné le terrain aux religieuses à la condition que les anglophones puissent avoir une classe anglaise au couvent ${ }^{148}$.

Ce bref aperçu des valeurs «seigneuriales» que nous avons cru déceler à partir des témoignages recueillis ne saurait être complet. Certes, il est coloré par le cadre d'analyse qui est le nôtre, c'est-à-dire celui de la quête des "persistances seigneuriales » au Québec. Il va de soi que ces individus nous ont parlé de ce qui nous intéressait, ont répondu aux questions qui leur ont été posées. Toutefois, ils ont souvent été bien au-devant de nos interrogations. Certains témoins se sont livrés, à partir de notre seule mise en situation, pendant de longues minutes, allant très souvent exactement où nous souhaitions les conduire. Néanmoins, ils nous ont aussi parfois menés ailleurs, mettant de l'avant des "valeurs» ou des traits familiaux qui dépassent la dimension seigneuriale : la tradition musicale remontant à Archibald Campbell au $\mathrm{Bic}^{149}$, l'importance de l'éducation pour la famille Masson, les imposantes figures littéraires que sont Anne Hébert et De Saint-Denys Garneau à Sainte-Catherine ou encore les relations entre anglophones et francophones en Beauce, autant de facettes qui viennent colorer l'histoire de ces familles d'ascendance seigneuriale dont chacune mériterait une analyse approfondie.

Que reste-t-il de la mémoire familiale/seigneuriale dans les localités concernées? Voilà ce que nous leur avons demandé en concluant. «Peu de choses» ont regretté certains qui voyaient dans notre enquête, justement, une manière de contribuer à assurer cette mémoire. Plusieurs ont déploré le peu d'intérêt pour l'histoire et le patrimoine de la part des politiciens municipaux ou des nouvelles générations. D’autres semblaient plus sereins quant à l'avenir, assumant le fait que la tradition seigneuriale appartient à

148. Entretien 2017-06 avec Gordon Pozer, Saint-Georges-de-Beauce, $1^{\text {er }}$ août 2017.

149. C'est d'ailleurs par l'interprétation d'un chant traditionnel écossais, tiré du livre de chants de son arrière-arrière-grand-mère Grace Campbell, que Jessica Cann a tenu à nous partager son héritage familial après le tournage de l'entretien de son père, Douglas Cann. Entretien 2015-11 avec Jessica Cann, Le Bic, 7 août 2015. Ce magnifique moment, capté sur caméra, conclut le moyen-métrage réalisé par Stéphanie Lanthier: Persistances et mémoires seigneuriales au Québec : la mémoire familiale (2016) : <https:// youtu.be/ufqvzV9e00g>. 
un passé pas si lointain mais à présent bien révolu. Enfin, dans le cas très singulier de Vaudreuil, 25 années de Seigneuriales donnent bon espoir à la famille quant à la perpétuation de son histoire.

\section{CONCLUSION}

L'histoire nous avait beaucoup plu. Elle flattait quelquesuns de nos mythes les plus chers: la terre et les pierres, la permanence, la résurrection du passé, l'éternel retour des temps ${ }^{150}$.

Nous avons esquissé dans ce texte quelques réflexions qui ressortent des entretiens réalisés auprès de descendants de seigneurs et dont l'analyse donnera lieu, nous l'espérons, à une monographie plus substantielle. Certains constats surgissent des entretiens et voient se dessiner une culture familiale imprégnée de ces vestiges d’Ancien Régime. Cette culture familiale se manifeste par une identité fortement ancrée au terroir ancestral, par une conscience de soi dans la longue durée et par des valeurs qui participent à l'idéal nobiliaire canadien: honneur, sens de l'engagement, bilinguisme...

Les entretiens révèlent aussi assez nettement une rupture située autour des années 1950-1960, que la plupart attribuent aux changements sociaux de la Révolution tranquille. Ces manières d'être deviendraient alors désuètes, parce qu'elles seraient inadaptées à une société égalitaire et fondée sur l'éducation plutôt que la naissance. Les individus nés après 1950 ont tous mis de l'avant cette rupture entre leur génération et celle de leurs parents, en termes de statut social et de rapports aux autres. Pour Amélie Masson-Labonté, 33 ans, rencontrée dans la seigneurie de ses ancêtres, Terrebonne, c'est un héritage qui fut pleinement assumé par son grand-père, lourd à porter pour sa mère et plutôt une "curiosité» pour elle-même ${ }^{151}$. Il faudra creuser ce «fossé des générations», en particulier dans les cas où nous avons eu accès à plusieurs membres d'une même famille appartenant à des générations différentes (Rioux, LotbinièreHarwood, Masson). Il conviendra aussi de porter une attention particulière à la dimension du genre, sur laquelle il n'a pas été possible de nous arrêter dans ces pages, en la croisant avec le facteur générationnel afin de vérifier s’il existe une mémoire seigneuriale "genrée», dans la filiation des

150. Jean d'Ormesson, Au plaisir de Dieu (Paris, Gallimard, 1974), p. 203.

151. Entretien 2016-06 avec Amélie Masson-Labonté, Terrebonne, 29 avril 2016. 
représentations stéréotypées du seigneur "paternel» et de la seigneuresse "charitable», qui semblent bien établies au XIX" siècle ${ }^{152}$.

Cette recherche, rappelons-le, ne vise pas à écrire l'histoire de ces familles ni de ces localités, mais plutôt à saisir leur mémoire telle que construite et représentée au terme d'une longue chaîne familiale. Bien qu'il faille être prudent face à ces témoignages en termes de représentativité, dans la mesure où ceux pour qui cette mémoire est inexistante ou insignifiante ne se sont pas montrés volontaires pour l'étude, c'est un biais qu'il faut accepter, comme dans toute étude d'histoire orale, avec ceci de particulier que le groupe à l'étude est bien petit. La mémoire seigneuriale que nous documentons concerne ceux et celles à qui «quelque chose» a été transmis. Ces individus révèlent, comme L.-A. Taschereau en 1922, des persistances d'Ancien Régime qui sont bien plus que folkloriques. Les reconstitutions historiques, comme les Seigneuriales de Vaudreuil-Dorion, les lieux patrimoniaux comme la Seigneurie des Aulnaies, voire le Regroupement des Anciennes Familles, une association regroupant les descendants de familles nobles ou seigneuriales - où l'on doit faire la preuve de sa généalogie pour adhérer, adoptent une perspective un peu nostalgique de l'histoire seigneuriale et peuvent porter à penser que cette histoire n'est plus que patrimoine et que l'abolition du régime seigneurial a mis un terme à ce mode de vie.

Pourtant, au moins jusque dans la première moitié $\mathrm{du} \mathrm{XX}^{\mathrm{e}}$ siècle, le prestige et l'autorité associés aux familles de l'ancienne aristocratie seigneuriale canadienne ont eu un impact à l'échelle locale et régionale, mais parfois aussi à l'échelle nationale. Que cette influence soit restée un fait négligé des historiens n'en fait pas pour autant un fait négligeable. Fernand Ouellet écrivait qu' "À la fin du XVIII" siècle la décadence des anciennes familles est suffisamment engagée pour que désormais elles ne puissent aspirer à jouer un rôle déterminant dans les structures politiques et sociales ${ }^{153}$. " À la lumière des présentes observations, cette affirmation nous apparaît peu convaincante ${ }^{154}$, invitant à de plus amples recherches afin d'étudier les mécanismes par lesquels ces familles se sont (ou pas) reproduites et maintenues, à l'instar des Taschereau, dans les sphères du pouvoir.

152. Dans un texte à paraître, nous invitons justement à approfondir cette question: Benoît Grenier, «Femmes et propriété seigneuriale au Canada ( $17^{\mathrm{e}}-19^{\mathrm{e}}$ siècles): Les formes de l'autorité des seigneuresses ", Histoire, Économie \& Société, sous presse.

153. Fernand Ouellet, «Le régime seigneurial dans le Québec (1760-1854)», dans Fernand Ouellet, Éléments d'histoire sociale du Bas-Canada (Montréal, Hurtubise HMH, 1972), p. 101.

154. Comme elle ne convainc pas non plus Brian Young, «Revisiting Feudal Vestiges... », p. 138. 